Article

\title{
Analysis of Entropy Generation Rate in an Unsteady Porous Channel Flow with Navier Slip and Convective Cooling
}

\section{Tirivanhu Chinyoka $^{1, *}$ and Oluwole Daniel Makinde ${ }^{2}$}

${ }^{1}$ Center for Research in Computational and Applied Mechanics, University of Cape Town, Rondebosch 7701, South Africa; E-Mail: tchinyok@vt.edu

${ }^{2}$ Institute for Advanced Research in Mathematical Modelling and Computations, Cape Peninsula University of Technology, P. O. Box 1906, Bellville 7535, South Africa;

E-Mail: makinded@cput.ac.za

* Author to whom correspondence should be addressed; E-Mail: tchinyok@vt.edu; Tel. +27-21-650-3198 Fax. +27-21-650-2334.

Received: 3 April 2013; in revised form: 6 May 2013 / Accepted: 21 May 2013 /

Published: 28 May 2013

\begin{abstract}
This study deals with the combined effects of Navier Slip, Convective cooling, variable viscosity, and suction/injection on the entropy generation rate in an unsteady flow of an incompressible viscous fluid flowing through a channel with permeable walls. The model equations for momentum and energy balance are solved numerically using semi-discretization finite difference techniques. Both the velocity and temperature profiles are obtained and utilized to compute the entropy generation number. The effects of key parameters on the fluid velocity, temperature, entropy generation rate and Bejan number are depicted graphically and analyzed in detail.
\end{abstract}

Keywords: permeable channel wall; convective cooling; variable viscosity; Navier slip; entropy generation; Bejan number

\section{Introduction}

The cornerstone in the field of heat transfer and thermal design is the second law analysis and its design-related concept of entropy generation minimization. The foundation of knowledge of entropy production goes back to Clausius and Kelvin's studies on the irreversible aspects of the Second Law 
of Thermodynamics [1-4]. Since then the theories based on these foundations have rapidly developed. However, the entropy production resulting from combined effects of velocity and temperature gradients has remained untreated by classical thermodynamics, which has motivated many researchers to conduct analyses of fundamental and applied engineering problems based on second law analyses. Entropy generation is associated with thermodynamic irreversibility, which is common in all types of heat transfer processes, namely conduction, convection and radiation. In thermodynamical analysis, the fundamental principle remains the improvement of the relevant thermal systems to mitigate against energy losses and hence fully optimize the energy resources. Since the pioneering work of Bejan [1-4], widespread research has been conducted on entropy generation analysis [5-7].

The work of Chinyoka [8] introduced uniform suction/injection at the walls as a means of mitigating the thermal effects of highly exothermic, reactive viscoelastic fluids. Our current work demonstrates that such an intervention also works in reducing heat generation in unsteady porous channel flows with Navier slip and convective cooling. Similarly, the work of Ikenna and Chinyoka [9] investigated the effects of wall slip at the channel walls on the heat transfer characteristics in reactive viscoelastic flows. It was shown in [9] that presence of wall slip enhanced heat build-up within the channel flow; our current results lead to similar conclusions.

The pioneering works of Aziz [10], Makinde and Aziz [11], and the references therein introduced the idea of using the convective boundary conditions in boundary layer flows. We adopt such boundary conditions in the current work. Makinde [12,13] investigated the thermal effects of reactive viscous flows through channels filled with porous media under isothermal wall conditions. As noted, several studies involving heat and mass transfer in Newtonian and non-Newtonian fluids [14-16] have been conducted but most lack a systematic and rational treatment of the thermodynamics of the problem with respect to the combined effects of porous walls, wall slip, unsteadiness, variable viscosity and asymmetric convective boundary conditions on the flow system.

The objective of the present work is to study the unsteady flow of a reactive variable viscosity fluid between two parallel porous plates acted upon by nonconstant pressure. Both the lower and upper walls of the channel are subjected to asymmetric convective heat exchange with the ambient and allow for uniform suction/injection in the transverse direction. The mathematical formulation of the problem is established in Section 2. In Section 3 the semi-implicit finite difference technique is implemented for the solution process of the coupled nonlinear problem. Graphical results are presented and discussed qualitatively and quantitatively with respect to various parameters embedded in the system in Section 4.

\section{Mathematical Model}

We consider unsteady flow of an incompressible viscous fluid through a channel with permeable walls, see Figure 1. It is assumed that the fluid is injected uniformly at a constant rate into the channel at the lower wall and fluid suction occurs at the upper wall at the same rate. 
Figure 1. Schematic diagram of the problem.

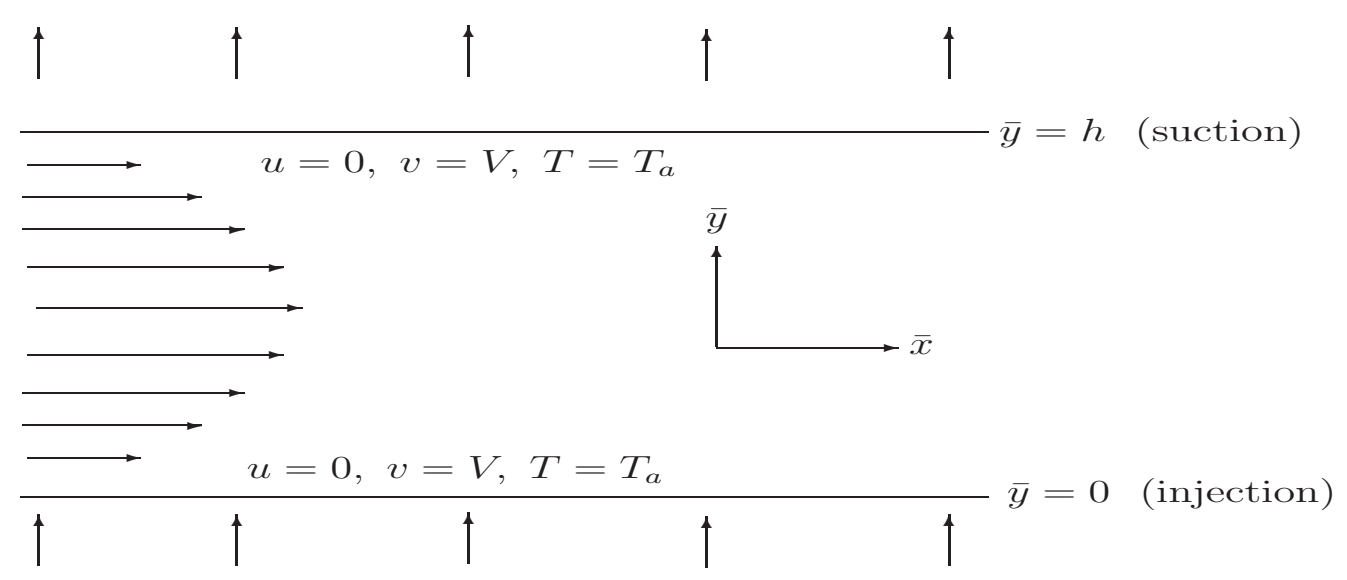

Under these assumptions, the governing equations for the momentum and energy balance in one dimension can be written as follows:

$$
\rho\left(\frac{\partial u}{\partial \bar{t}}+V \frac{\partial u}{\partial \bar{y}}\right)=-\frac{\partial \bar{P}}{\partial \bar{x}}+\frac{\partial}{\partial \bar{y}}\left(\bar{\mu}(T) \frac{\partial u}{\partial \bar{y}}\right)
$$

and

$$
\rho c_{p}\left(\frac{\partial T}{\partial \bar{t}}+V \frac{\partial T}{\partial \bar{y}}\right)=k \frac{\partial^{2} T}{\partial \bar{y}^{2}}+\bar{\mu}(T)\left(\frac{\partial u}{\partial \bar{y}}\right)^{2}
$$

The appropriate initial and boundary conditions are:

$$
\begin{aligned}
& u(\bar{y}, 0)=0, \quad T(\bar{y}, 0)=T_{0} \\
& \beta_{1} u(0, \bar{t})=\bar{\mu}(T) \frac{\partial u}{\partial \bar{y}}(0, \bar{t}), \quad-k \frac{\partial T}{\partial \bar{y}}(0, \bar{t})=\gamma_{1}\left[T_{a}-T(0, \bar{t})\right] \\
& \beta_{2} u(h, \bar{t})=\bar{\mu}(T) \frac{\partial u}{\partial \bar{y}}(h, \bar{t}), \quad-k \frac{\partial T}{\partial \bar{y}}(0, \bar{t})=\gamma_{2}\left[T(h, \bar{t})-T_{a}\right]
\end{aligned}
$$

where $h$ is the channel width, $u$ is the velocity of the fluid, $P$ is the fluid pressure, $V$ is the uniform suction/injection velocity at the channel walls, $k$ is the thermal conductivity coefficient, $\rho$ is the fluid density, $c_{p}$ is the specific heat at constant pressure, $1 \gamma_{1}$ is the lower wall heat transfer coefficient, $\gamma_{2}$ is the upper wall heat transfer coefficient, $\beta_{1}$ is the lower wall slip parameter, $\beta_{2}$ is the upper wall slip parameter, $T$ is the temperature and $T_{a}$ is the ambient temperature. The temperature dependant viscosity $(\bar{\mu})$ can be expressed as

$$
\bar{\mu}(T)=\mu_{0} e^{-b\left(T-T_{0}\right)}
$$


where $b$ is a viscosity variation parameter and $\mu_{0}$ is the initial fluid dynamic viscosity at temperature $T_{0}$. We introducing the following non-dimensional quantities:

$$
\begin{aligned}
& \eta=\frac{\bar{y}}{h}, t=\frac{V \bar{t}}{h}, X=\frac{\bar{x}}{a}, W=\frac{u}{V}, \theta=\frac{T-T_{0}}{T_{a}-T_{0}}, P=\frac{\bar{P} h}{\mu_{0} V} \\
& \operatorname{Re}=\frac{\rho h V}{\mu_{0}}, \operatorname{Pr}=\frac{\mu_{0} c_{p}}{k}, \mathrm{Bi}_{1}=\frac{\gamma_{1} h}{k}, \mathrm{Bi}_{2}=\frac{\gamma_{2} h}{k}, G=-\frac{\partial P}{\partial X} \\
& \mu=\frac{\bar{\mu}}{\mu_{0}}, m=b\left(T_{a}-T_{0}\right), \lambda_{1}=\frac{\mu_{0}}{h \beta_{1}}, \lambda_{2}=\frac{\mu_{0}}{h \beta_{2}}, \mathrm{Ec}=\frac{V^{2}}{c_{p}\left(T_{a}-T_{0}\right)}
\end{aligned}
$$

Substituting Equation (8) into Equations (1)-(6), we obtain

$$
\begin{aligned}
& \operatorname{Re}\left(\frac{\partial W}{\partial t}+\frac{\partial W}{\partial \eta}\right)=G+e^{-m \theta} \frac{\partial^{2} W}{\partial \eta^{2}}-m e^{-m \theta} \frac{\partial \theta}{\partial \eta} \frac{\partial W}{\partial \eta} \\
& \operatorname{Re} \operatorname{Pr}\left(\frac{\partial \theta}{\partial t}+\frac{\partial \theta}{\partial \eta}\right)=\frac{\partial^{2} \theta}{\partial \eta^{2}}+\operatorname{Ec} \operatorname{Pr}\left(\frac{\partial W}{\partial \eta}\right)^{2}
\end{aligned}
$$

with the initial and boundary conditions given as

$$
\begin{aligned}
& W(\eta, 0)=0, \quad \theta(\eta, 0)=0 \\
& W(0, t)=\lambda_{1} e^{-m \theta(0, t)} \frac{\partial W}{\partial \eta}(0, t), \quad \frac{\partial \theta}{\partial \eta}(0, t)=\mathrm{Bi}_{1}[\theta(0, t)-1] \\
& W(1, t)=\lambda_{2} e^{-m \theta(1, t)} \frac{\partial W}{\partial \eta}(1, t), \quad \frac{\partial \theta}{\partial \eta}(1, t)=\mathrm{Bi}_{2}[1-\theta(1, t)]
\end{aligned}
$$

where $m$ is the viscosity variation parameter, $\lambda_{1}$ is the lower wall slip parameter, $\lambda_{2}$ is the upper wall slip parameter, $G$ is the pressure gradient parameter, Re is the Reynolds number, Pr is the Prandtl number, Ec is the Eckert number, $\mathrm{Bi}_{1}$ is the lower wall Biot number, and $\mathrm{Bi}_{2}$ is the upper wall Biot number.

In the Section 4, the coupled nonlinear boundary value problem represented by Equations (8)-(9) together with their initial and boundary conditions (10)-(12) are solved numerically using semi-discretization finite difference techniques.

\section{Entropy Analysis}

Following Bejan [1], Mahmud and Fraser [7], the local entropy generation rate for a viscous incompressible fluid is given as

$$
E_{G}=\frac{k}{T_{0}^{2}}\left(\frac{\partial T}{\partial \bar{y}}\right)+\frac{\bar{\mu}}{T_{0}}\left(\frac{\partial \bar{u}}{\partial \bar{y}}\right)^{2}
$$

The first term in Equation (13) is the irreversibility due to heat transfer and the second term is the entropy generation due to viscous dissipation and third term is due to magnetic field. In dimensionless form, Equation (13) becomes,

$$
\mathrm{Ns}=\frac{h^{2} T_{0}^{2} E_{G}}{k\left(T_{a}-T_{0}\right)^{2}}=\left(\frac{\partial \theta}{\partial \eta}\right)^{2}+\frac{\operatorname{Br} e^{-m \theta}}{\Omega}\left(\frac{\partial W}{\partial \eta}\right)^{2}
$$


where $\Omega=\left(T_{a}-T_{0}\right) / T_{0}$ is the temperature difference parameter and $\mathrm{Br}=\mathrm{Ec} \operatorname{Pr}$ is the Brinkmann number. Let

$$
N_{1}=\left(\frac{\partial \theta}{\partial r}\right)^{2}, \quad N_{2}=\frac{\operatorname{Br} e^{-m \theta}}{\Omega}\left(\frac{\partial u}{\partial r}\right)^{2}, \quad \Phi=\frac{N_{2}}{N_{1}}
$$

We then define the Bejan number as

$$
\mathrm{Be}=\frac{N_{1}}{N_{s}}=\frac{1}{1+\Phi}
$$

where $\Phi$ is the irreversibility ratio. Bejan number ranges from 0 to 1 . The limit of fluid friction irreversibility dominant effects is given by $\mathrm{Be}=0$, while $\mathrm{Be}=1$ is the limit where the irreversibility due to heat transfer dominates the flow system.

\section{Numerical Solution}

Our numerical algorithm is based on the semi-implicit finite difference scheme [8,9,17-20]. Implicit terms are taken at the intermediate time level $(N+\xi)$ where $0 \leq \xi \leq 1$.

The discretization of the governing equations is based on a linear Cartesian mesh and uniform grid on which finite-differences are taken. We approximate both the second and first spatial derivatives with second-order central differences.

The equations corresponding to the first and last grid points are modified to incorporate the boundary conditions. The semi-implicit scheme for the velocity component reads:

$$
\operatorname{Re}\left(\frac{\partial W}{\partial t}+\frac{\partial}{\partial \eta} W^{(N)}\right)=G+e^{-m \theta^{(N)}} \frac{\partial^{2}}{\partial \eta^{2}} W^{(N+\xi)}+\left[m e^{-m \theta} \frac{\partial \theta}{\partial \eta} \frac{\partial W}{\partial \eta}\right]^{(N)}
$$

In Equation (17) it is understood that $\partial \# / \partial t:=\left(\#^{(N+1)}-\#^{(N)}\right) / \Delta t$. The equation for $W^{(N+1)}$ then becomes:

$$
-r_{1} W_{j-1}^{(N+1)}+\left(\operatorname{Re}+2 r_{1}\right) W_{j}^{(N+1)}-r_{1} W_{j+1}^{(N+1)}=\text { explicit terms }
$$

where

$$
r_{1}=\xi \frac{\Delta t}{\Delta \eta^{2}} \exp (-m \theta)
$$

The solution procedure for $W^{(N+1)}$ thus reduces to inversion of tri-diagonal matrices, which is an advantage over a full implicit scheme. The semi-implicit integration scheme for the temperature equation is similar to that for the velocity component. Unmixed second partial derivatives of the temperature are treated implicitly:

$$
\operatorname{Re} \operatorname{Pr}\left(\frac{\partial \theta}{\partial t}+\frac{\partial}{\partial \eta} \theta^{(N)}\right)=\frac{\partial^{2}}{\partial \eta^{2}} \theta^{(N+\xi)}+\operatorname{Ec} \operatorname{Pr}\left(\frac{\partial}{\partial \eta} W^{(N)}\right)^{2}
$$

The equation for $\theta^{(N+1)}$ thus becomes:

$$
-r \theta_{j-1}^{(N+1)}+(\operatorname{Re} \operatorname{Pr}+2 r) \theta_{j}^{(N+1)}-r \theta_{j+1}^{(N+1)}=\text { explicit terms }
$$


where $r=\xi \Delta t / \Delta \eta^{2}$. The solution procedure again reduces to inversion of tri-diagonal matrices. The schemes (18 and 20) were checked for consistency. For $\xi=1$, these are first-order accurate in time but second order in space. The schemes in Chinyoka [19] have $\xi=1 / 2$, which improves the accuracy in time to second order. As in Chinyoka $[8,9,20]$ we, however, use $\xi=1$ here so that we are free to choose larger time steps and still converge to the steady solutions.

\section{Results and Discussion}

Unless otherwise stated, we employ the parameter values:

$G=1, \operatorname{Pr}=0.71, \lambda_{1}=0.1, \lambda_{2}=0.1, \mathrm{Bi}_{1}=0.1, \mathrm{Bi}_{2}=0.1 m=0.1, \Omega=0.1, \Delta \eta=0.01$, $\Delta t=0.01, \operatorname{Re}=0.1, t=50$.

These will be the default values in this work. In the succeeding graphics, if any of these parameter values is not explicitly mentioned, it will be understood that such parameters take on the default values.

\subsection{Transient and Steady Flow Profiles}

We display the transient solutions in Figure 2. The figures show a transient increase in both fluid velocity, Figure $2 a$, and temperature, Figure $2 b$, until a steady state is reached.

Figure 2. Transient and steady state profiles.

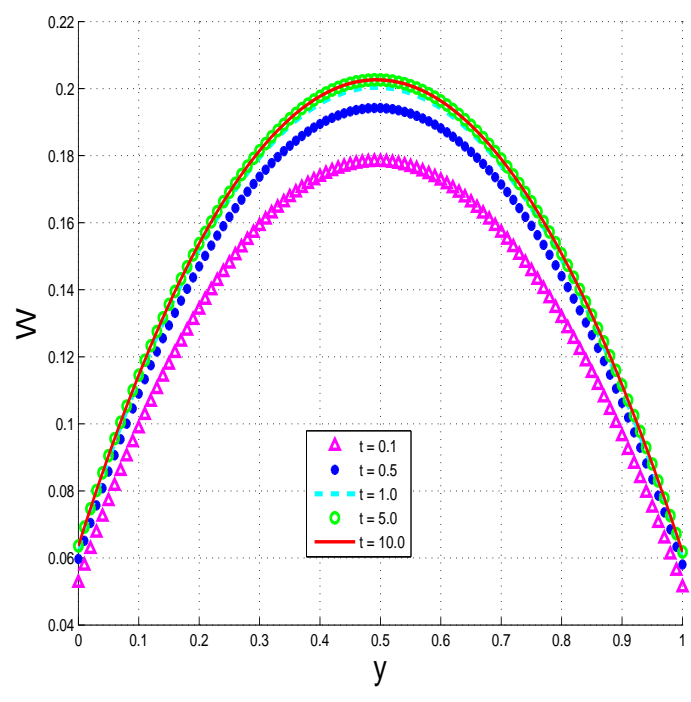

(a)

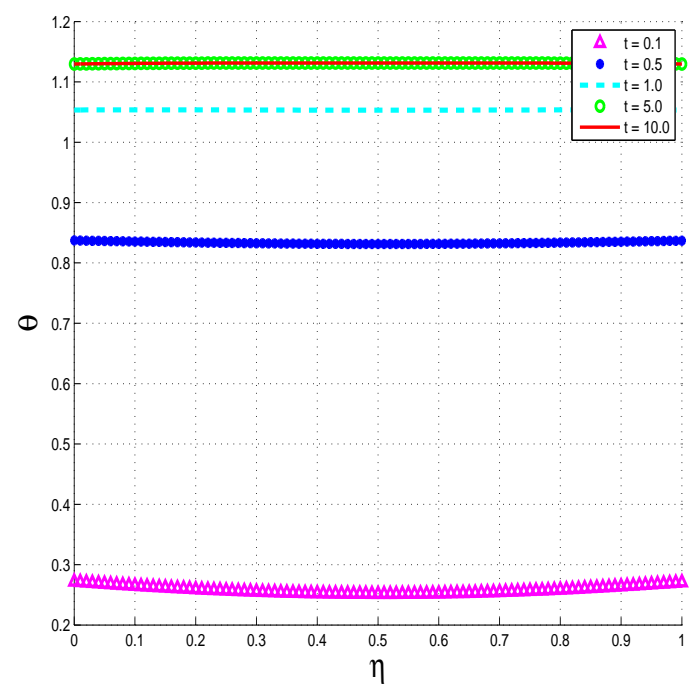

(b)

\subsection{Code Validation}

The steady flow case with $m=0$ is readily amenable to analytical treatment. We demonstrate in this section that our numerical scheme accurately reproduces this analytic solution. The steady equation for the velocity subject to $m=0$ is given by

$$
\operatorname{Re} \frac{d W}{d \eta}=G+\frac{d^{2} W}{d \eta^{2}}
$$


with boundary conditions given as

$$
W(0)=\lambda_{1} \frac{d W}{d \eta}(0), \quad W(1)=\lambda_{2} \frac{d W}{d \eta}(1)
$$

The analytic solution reads:

$$
W(\eta)=C_{1} \exp (\operatorname{Re} \eta)+\frac{G}{R e} \eta+C_{0}
$$

where for example,

$$
\begin{aligned}
C_{1} & =\frac{G\left(1+\lambda_{1}-\lambda_{2}\right)}{\operatorname{Re}\left(1-\exp (\mathrm{Re})+\lambda_{2} \operatorname{Re} \exp (\mathrm{Re})-\lambda_{1} \operatorname{Re}\right)} \\
C_{0} & =\lambda_{1}\left(C_{1} \operatorname{Re}+G / \operatorname{Re}\right)-C_{1}
\end{aligned}
$$

A comparison plot of the exact solution and the corresponding numerical solution (obtained at $t=10$ ) is given in Figure 3.

Figure 3. Analytic and numerical solution: (a) default values, (b) $\lambda_{1}=\lambda_{2}=0$.

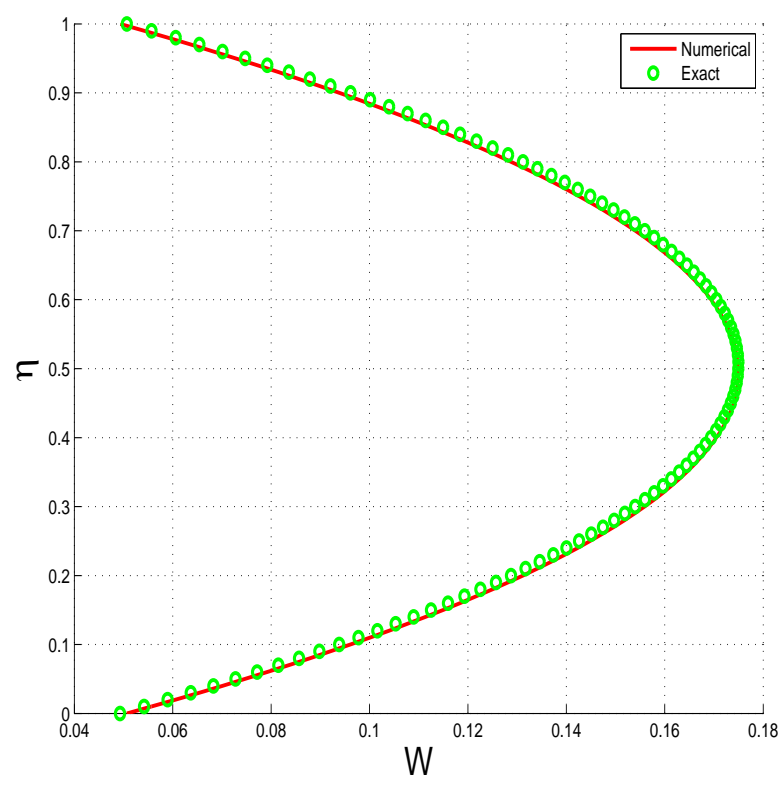

(a)

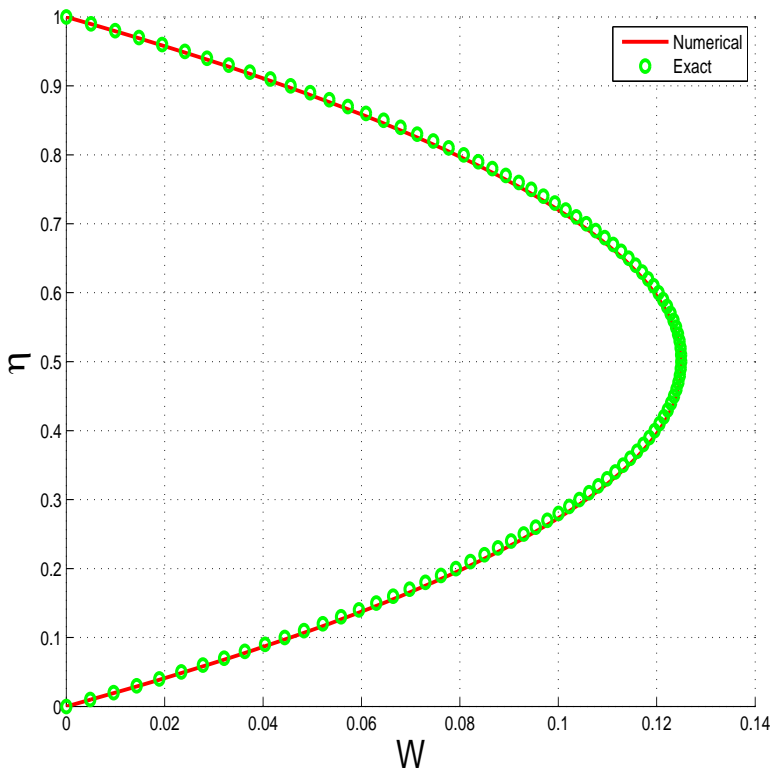

(b)

\subsubsection{Parameter Dependence of Solutions}

The response of the velocity and temperature to varying values of the Prandtl number $(\mathrm{Pr})$ is illustrated in Figure 4.

Larger values of the Prandtl number correspondingly increase the strength of the heat sources in the temperature equation and hence in turn increases the overall fluid temperature as clearly illustrated in Figure 4b. The increased temperature leads to decreased fluid viscosity and hence to higher fluid velocities as illustrated in Figure 4a. 
Figure 4. Effects of the Prandtl number, Pr.

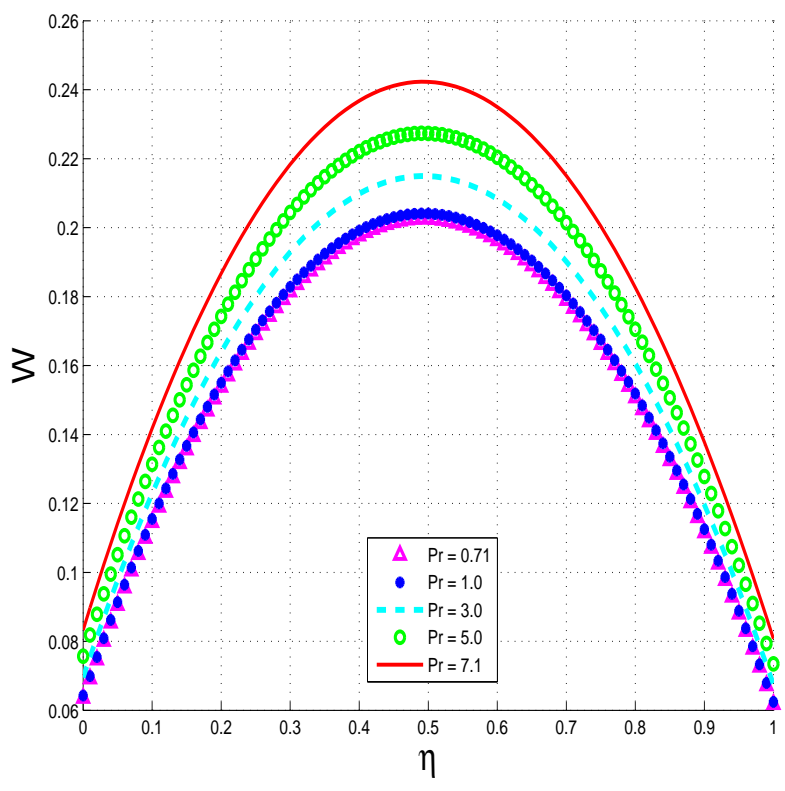

(a)

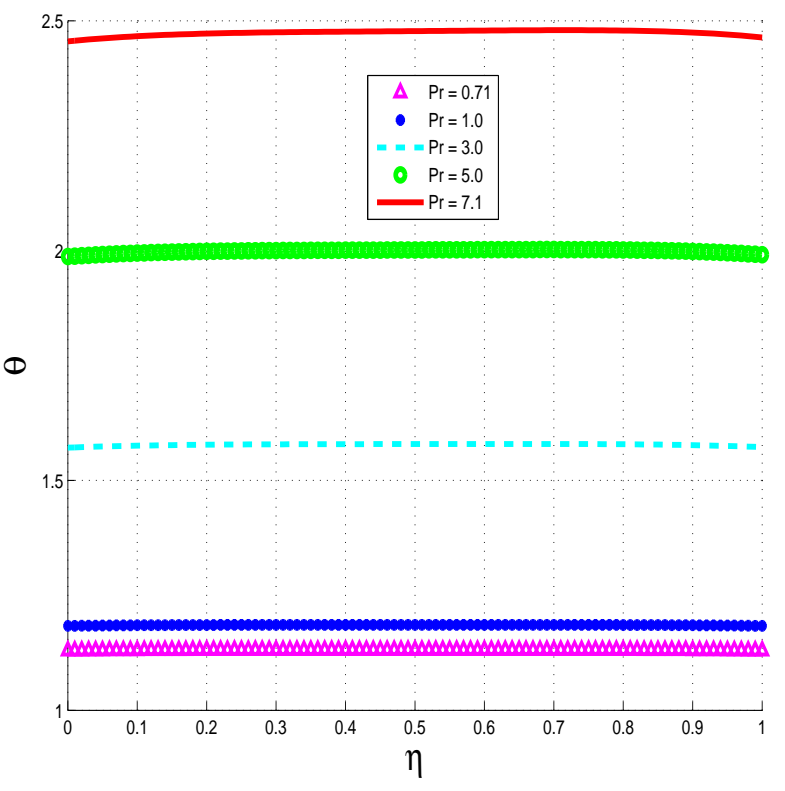

(b)

The response of the velocity and temperature to varying values of the Reynolds number $(\operatorname{Re})$ is illustrated in Figure 5.

Figure 5. Effects of the Reynolds number, Re.

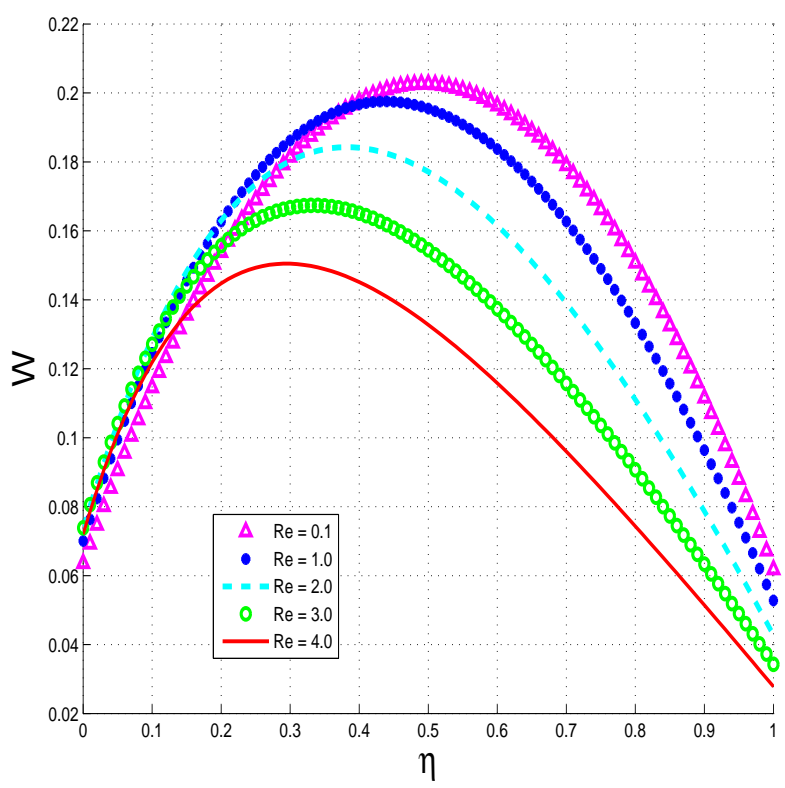

(a)

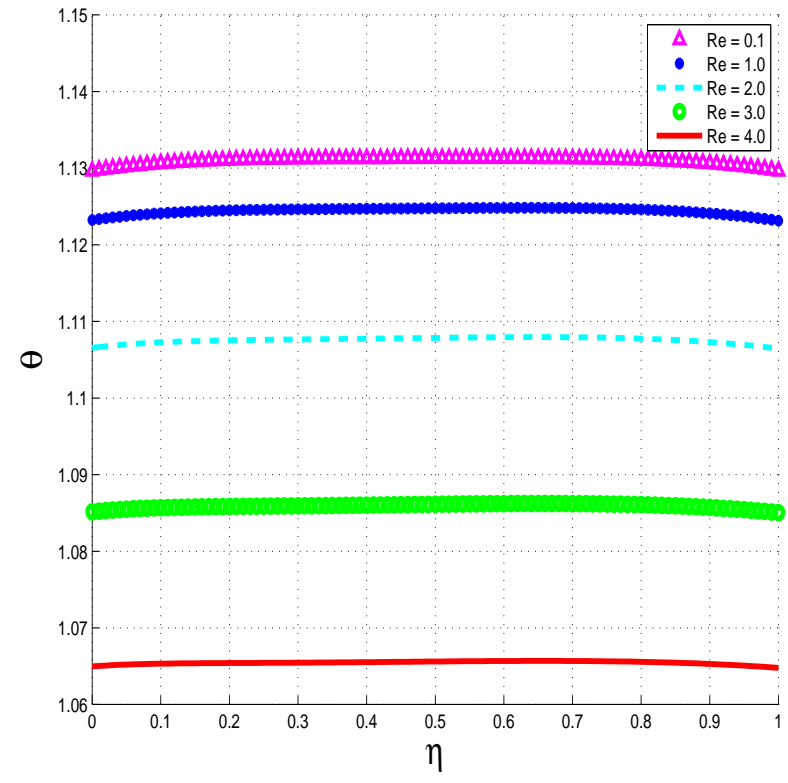

(b)

Larger values of the Reynolds number correspond to higher suction/injection strength and hence clearly decreases the axial fluid velocity as illustrated in Figure 5a. The decreased velocity leads to decreased heat source strength and hence to lower fluid temperature as illustrated in Figure 5b. The trends shown in Figures 4 and 5 in which the velocity and temperature either both increase or decrease 
together will be reproducible throughout our investigations and hence the explanations already given will not be repeated.

The response of the velocity and temperature to varying values of the viscosity parameter $(m)$ is illustrated in Figure 6.

Figure 6. Effects of the viscosity parameter, $m$.

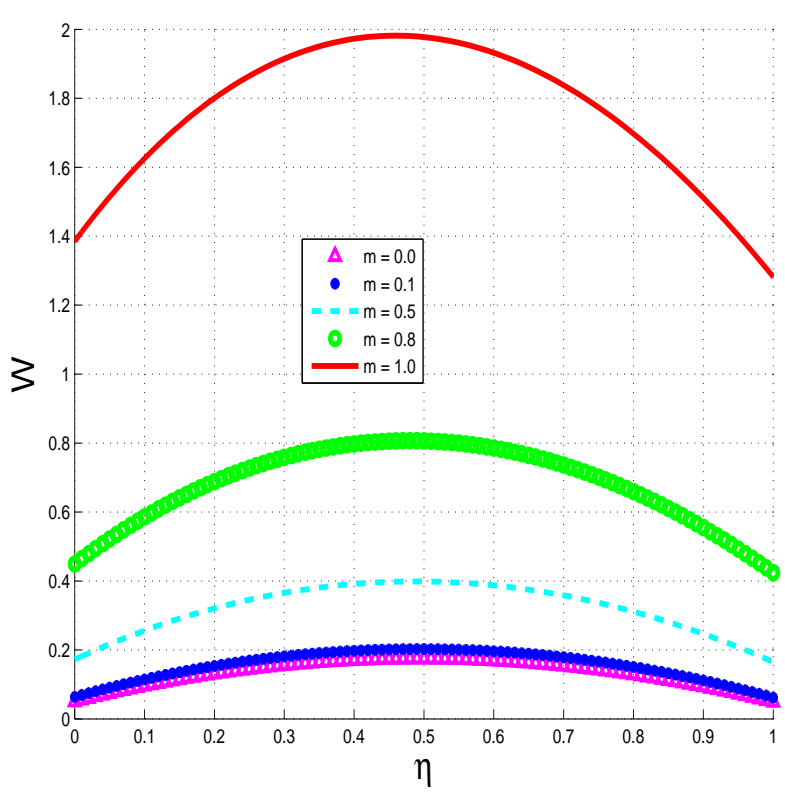

(a)

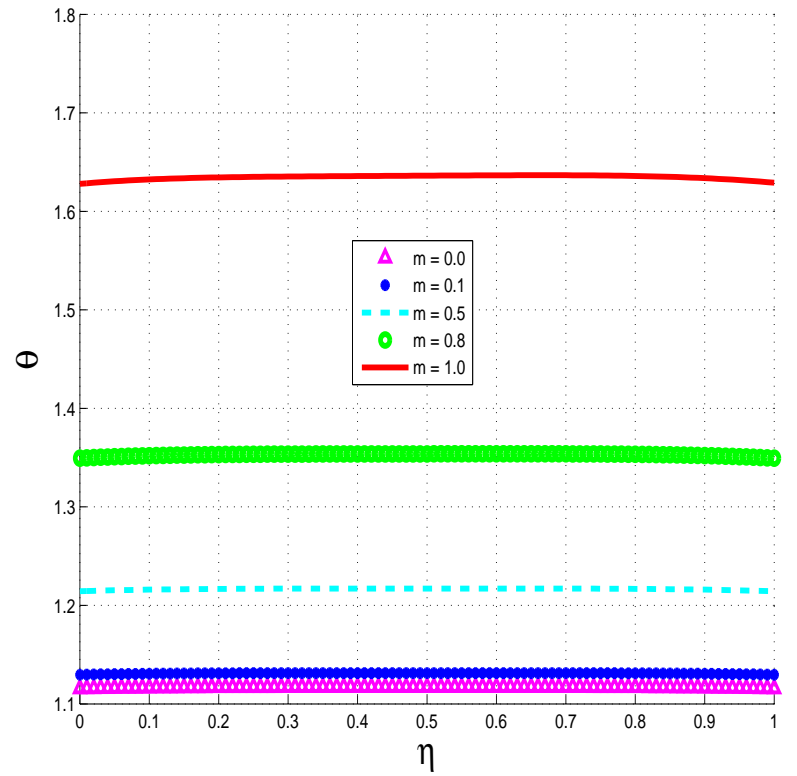

(b)

As expected, both velocity and temperature increase with increasing viscosity parameter (i.e., with decreasing fluid viscosity).

The effects of the wall Biot numbers on the velocity and temperature profiles is illustrated in Figures 7 and 8.

Figure 7. Effects of the lower wall Biot number $\left(\mathrm{Bi}_{1}\right)$.

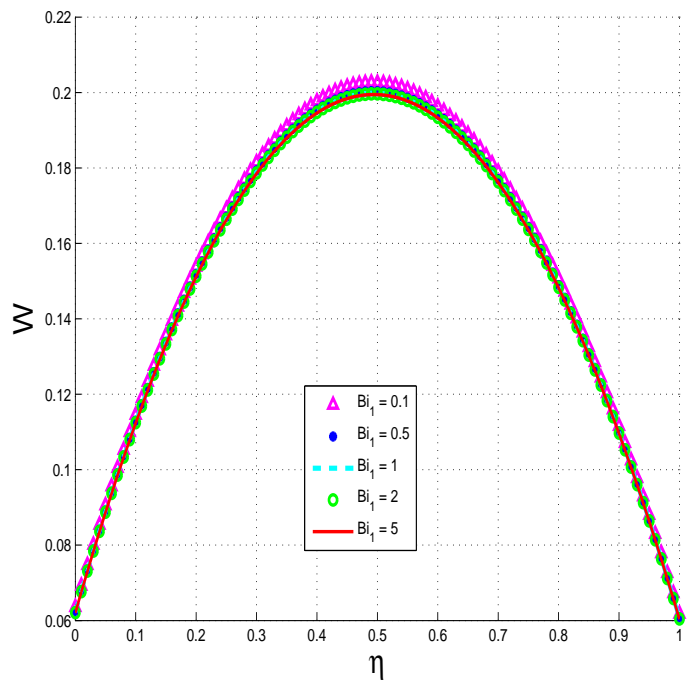

(a)

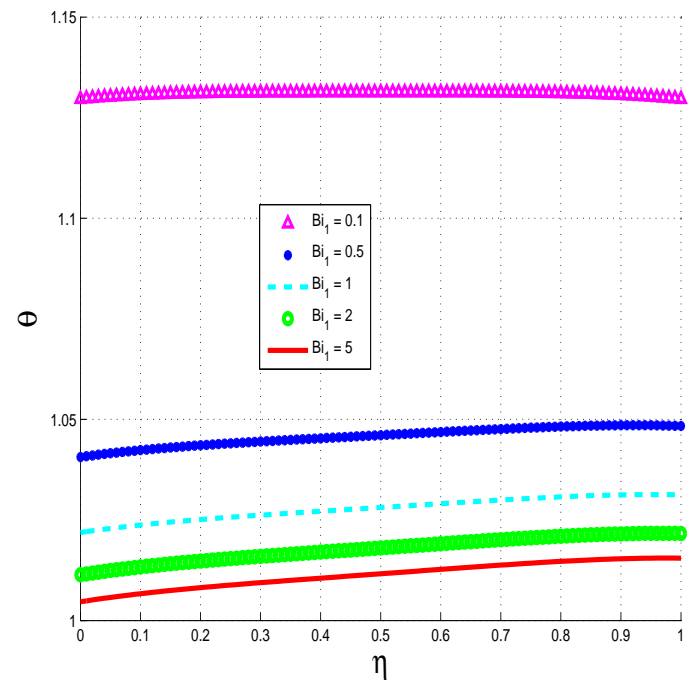

(b) 
Figure 8. Effects of the upper wall Biot number $\left(\mathrm{Bi}_{2}\right)$.

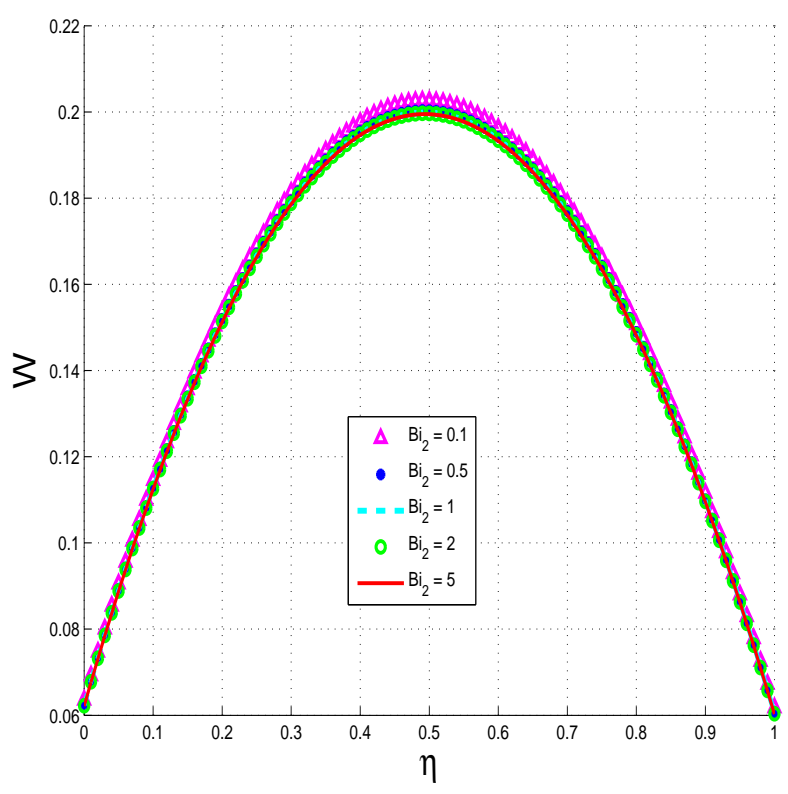

(a)

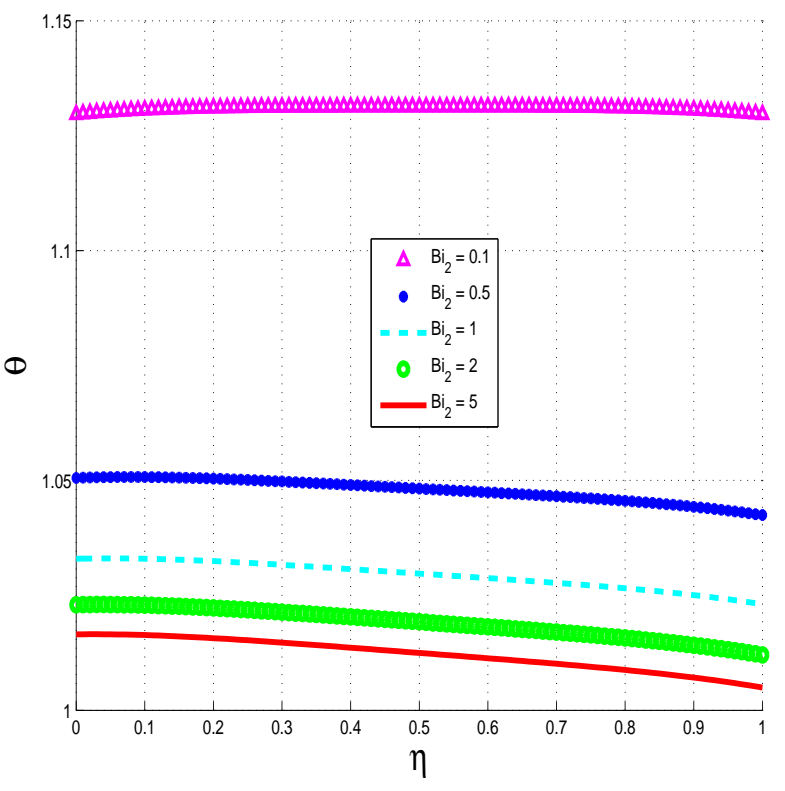

(b)

As seen from the temperature boundary conditions (12), higher Biot numbers mean correspondingly higher degrees of convective cooling at the channel walls, thus leading to lower temperatures at the channel walls and hence also in the bulk fluid. The overall temperature profiles thus decrease with increasing Biot number as the bulk fluid continually adjusts to the lower wall temperatures.

The response of the velocity and temperature to varying values of the Eckert number (Ec) is illustrated in Figure 9.

Figure 9. Effects of the Eckert number, (Ec).

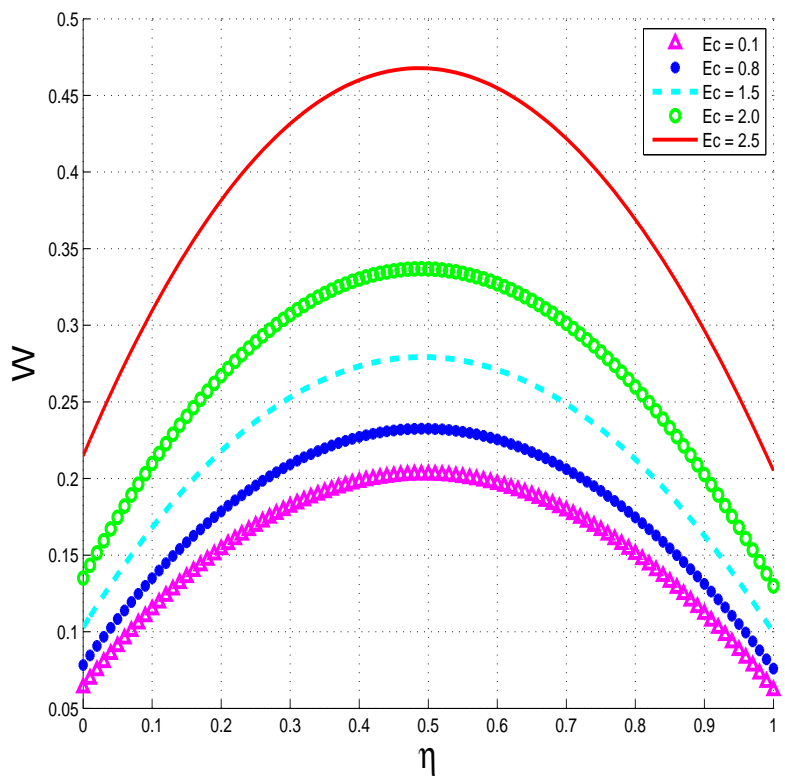

(a)

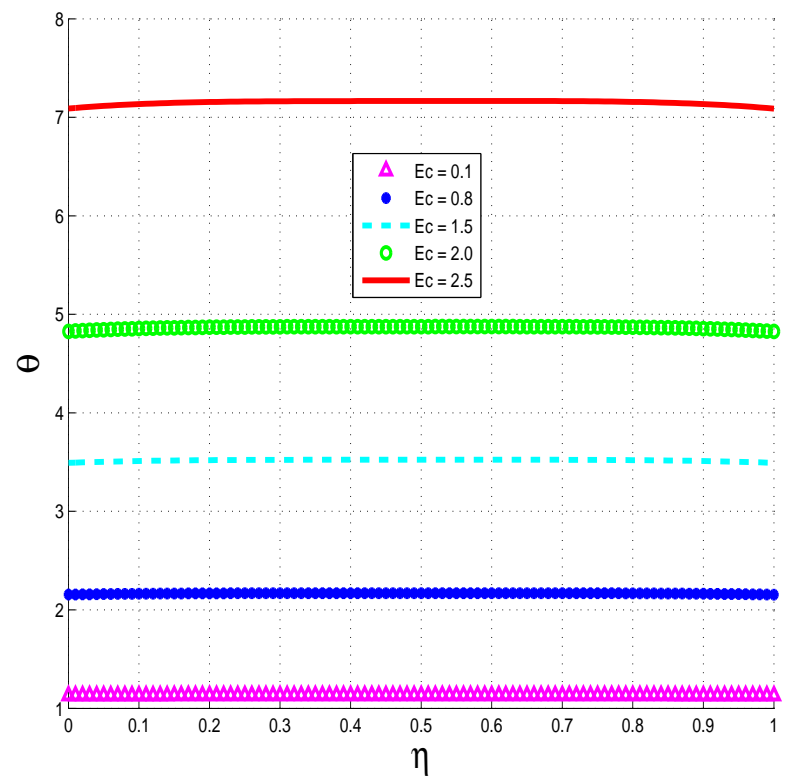

(b) 
The effects of the Eckert number are similar to those for the Prandtl number. The effects of the wall slip parameters on the velocity and temperature profiles is illustrated in Figures 10 and 11.

Figure 10. Effects of the lower wall slip parameter, $\left(\lambda_{1}\right)$.

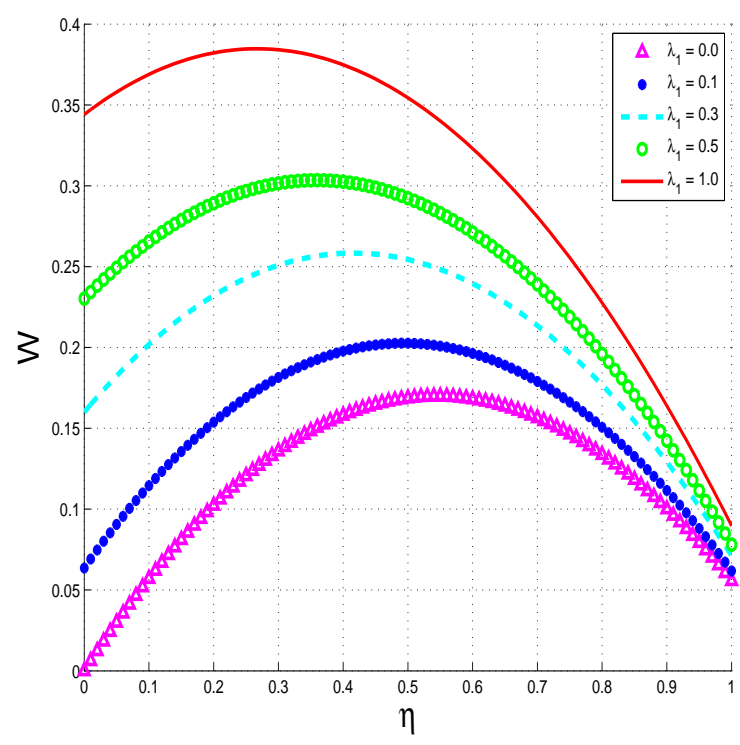

(a)

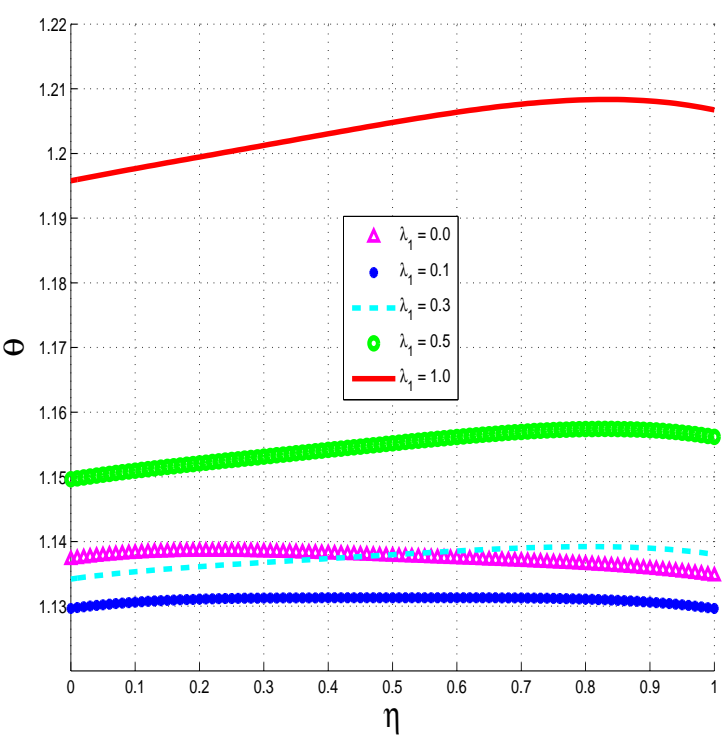

(b)

Figure 11. Effects of the upper wall slip parameter, $\left(\lambda_{2}\right)$.

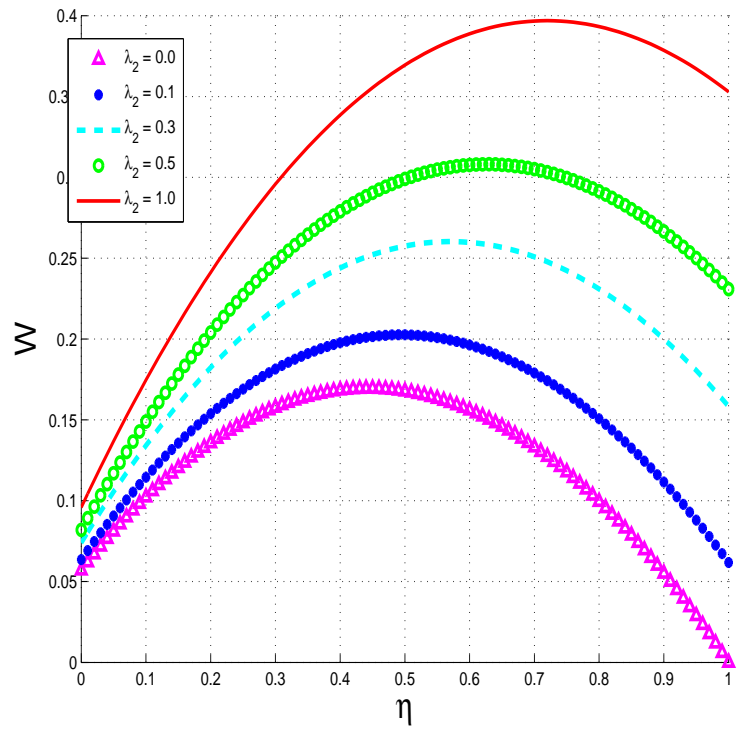

(a)

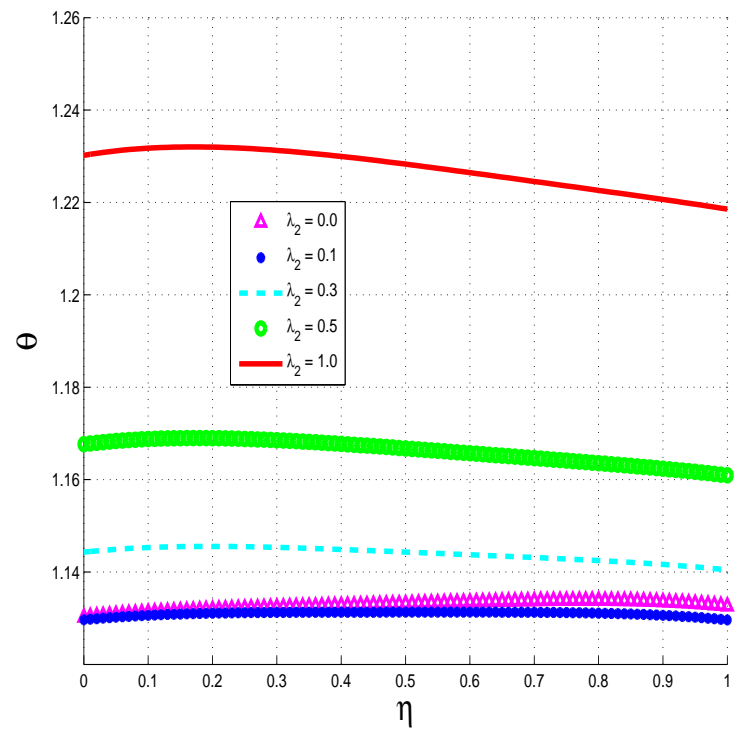

(b)

As expected, an increase in the slip parameters correspondingly increases the wall (and hence also the bulk) fluid velocity. 


\subsection{Entropy Generation}

In this section, we plot the entropy generation rate $(\mathrm{Ns})$ across the channel under varying parameter conditions. With the exception of the time evolution graphs shown in Figures 12 and 13, all other graphs are drawn at the time $t=10$.

Figure 12. Variation of entropy generation rate with $\eta$ and $t$.

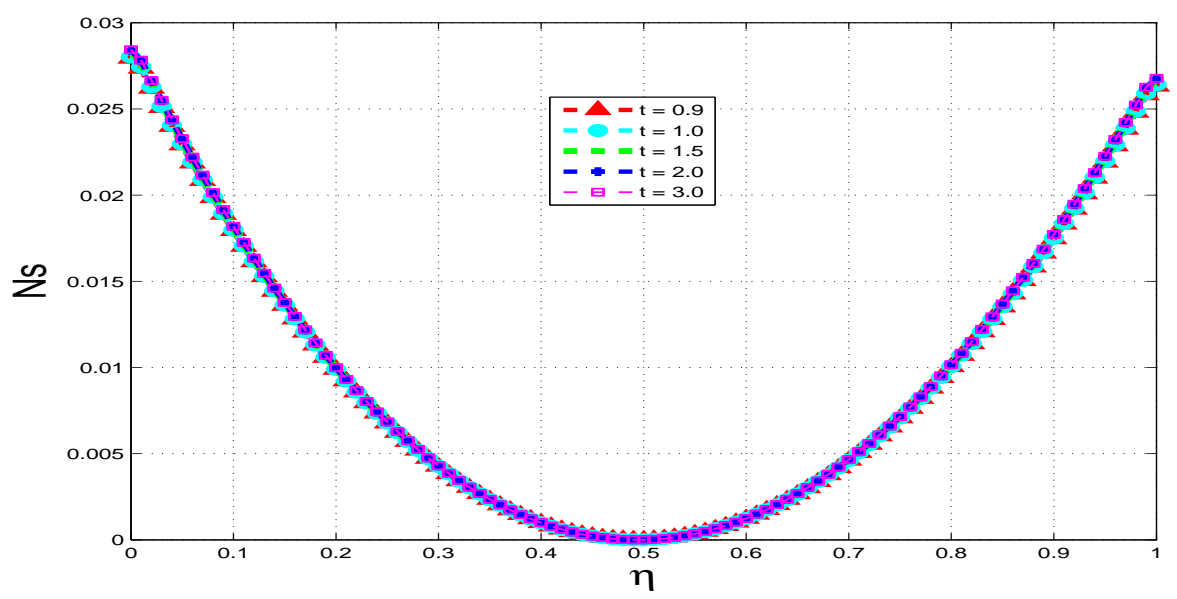

Figure 13. Variation of Bejan number with $\eta$ and $t$.

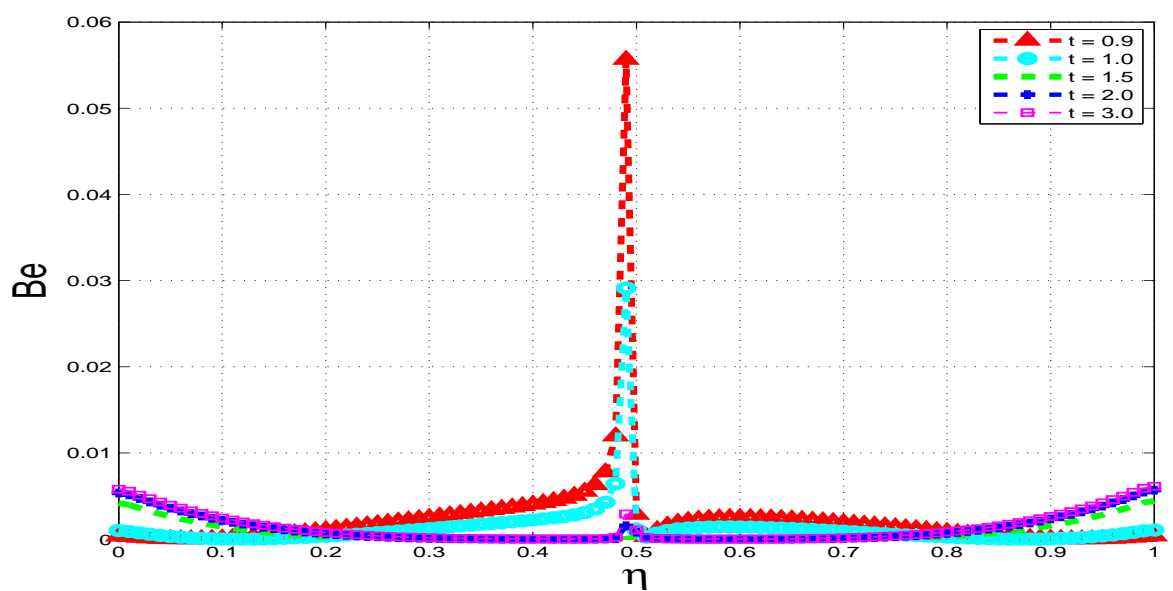

Figures 12-21 show the expected results for Ns. In particular, Ns clearly increases with $\operatorname{Br} \Omega^{-1}$, and decreases with increasing $m$. Parameters that increase the velocity and temperature gradients also increase the entropy generation rate and vice versa. In Figures 12-21, the entropy generation rate is expectedly maximum at the walls where velocity and temperature gradients as well as fluid viscosity are highest and minimum around the channel centerline where the maximum temperature and velocity and hence also zero temperature and velocity gradients are recorded. 
Figure 14. Variation of entropy generation rate with $\eta$ and Re.

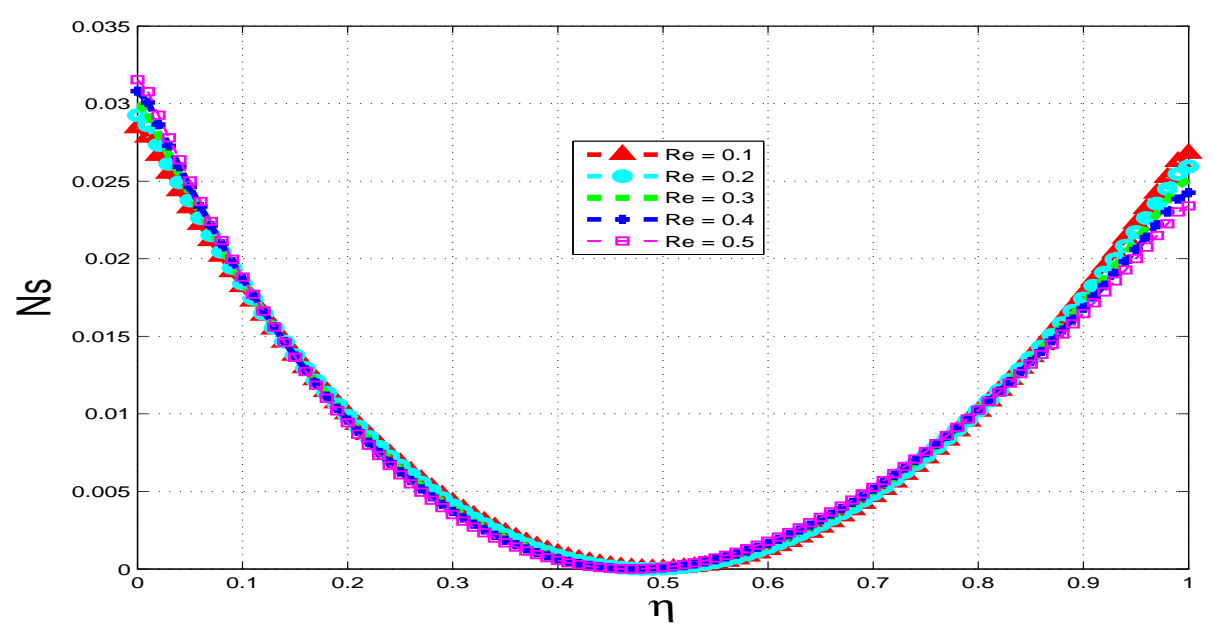

Figure 15. Variation of entropy generation rate with $\eta$ and $\operatorname{Pr}$.

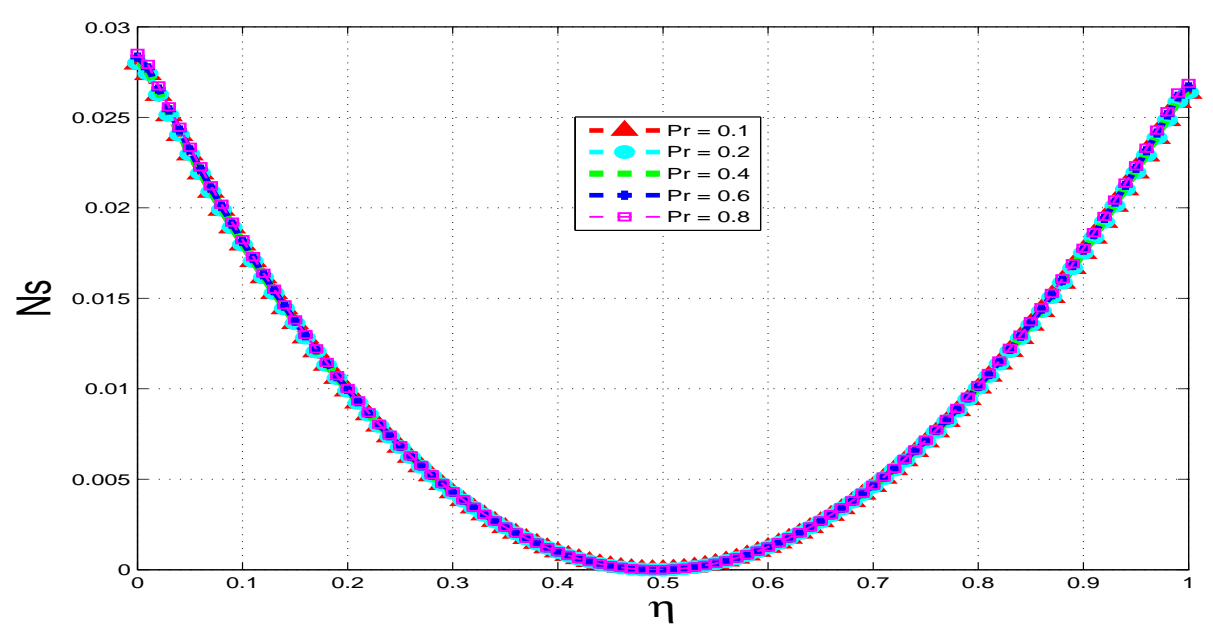

Figure 16. Variation of entropy generation rate with $\eta$ and $m$.

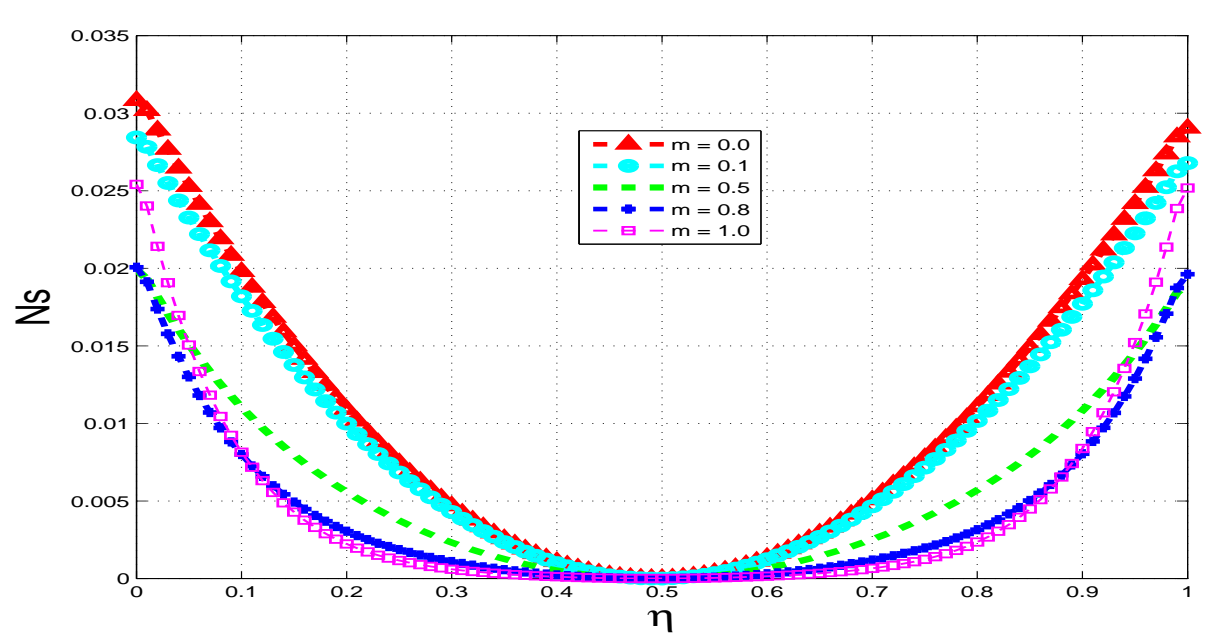


Figure 17. Variation of entropy generation rate with $\eta$ and $\operatorname{Br} \Omega^{-1}$.

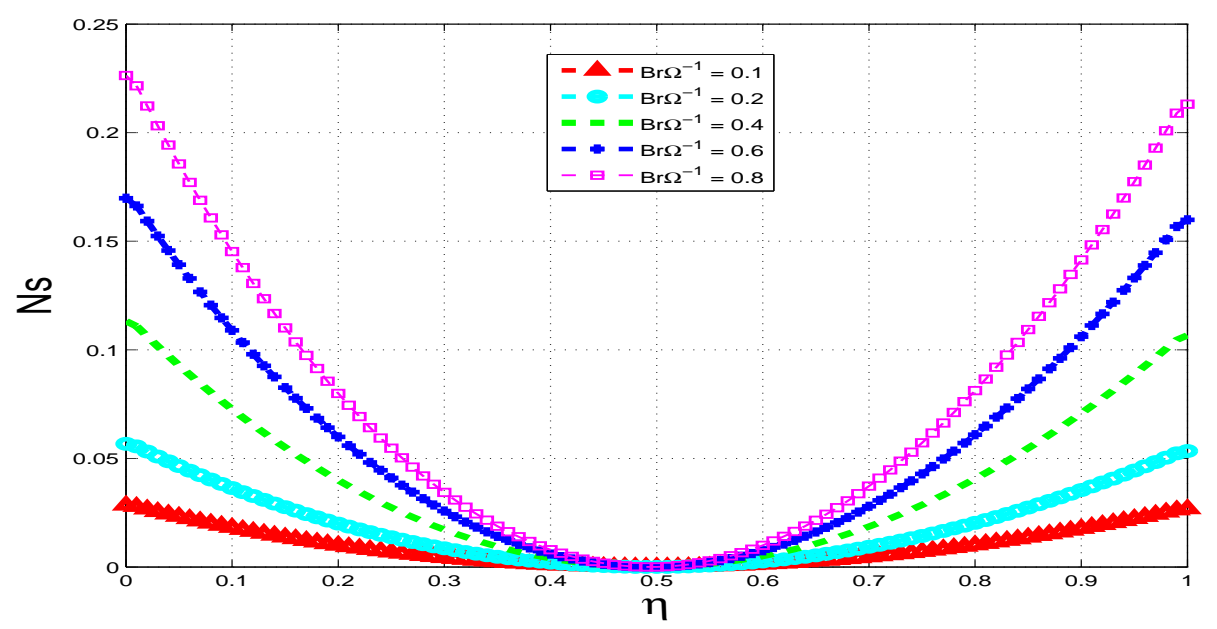

Figure 18. Variation of entropy generation rate with $\eta$ and $\lambda_{1}$.

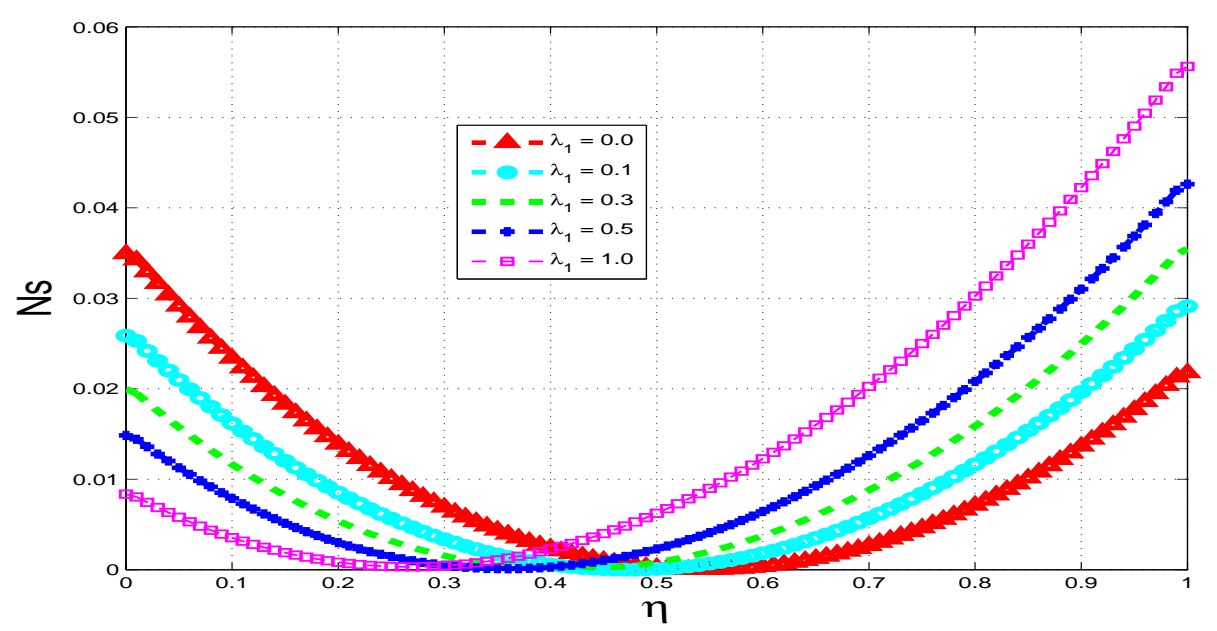

Figure 19. Variation of entropy generation rate with $\eta$ and $\lambda_{2}$.

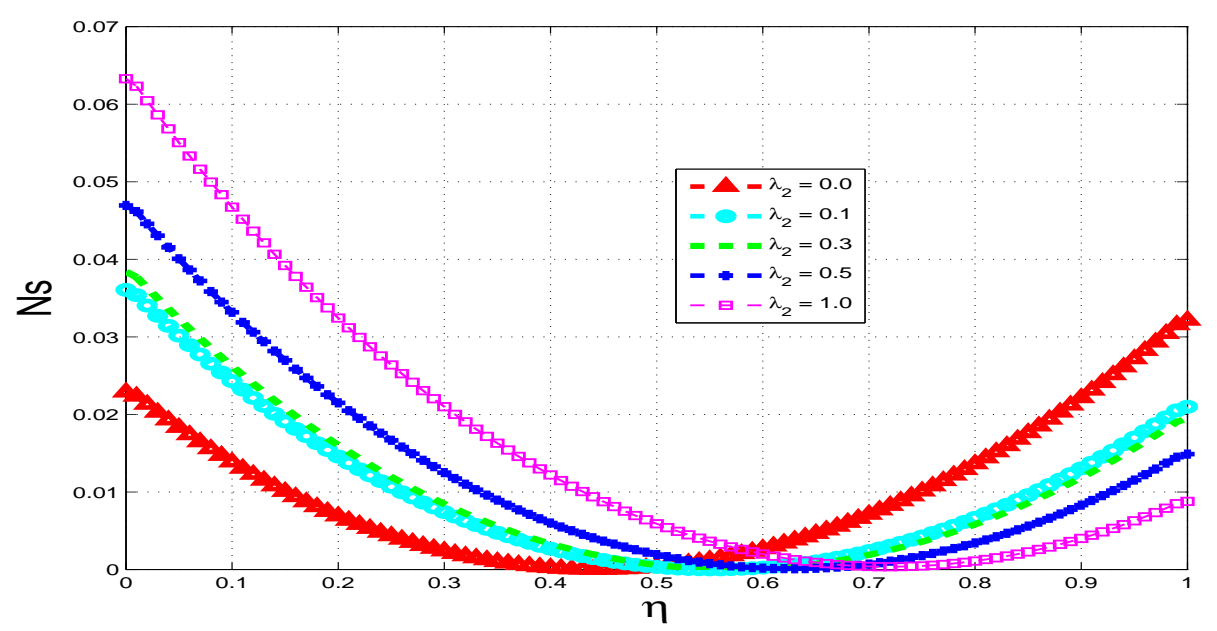


Figure 20. Variation of entropy generation rate with $\eta$ and $\mathrm{Bi}_{1}$.

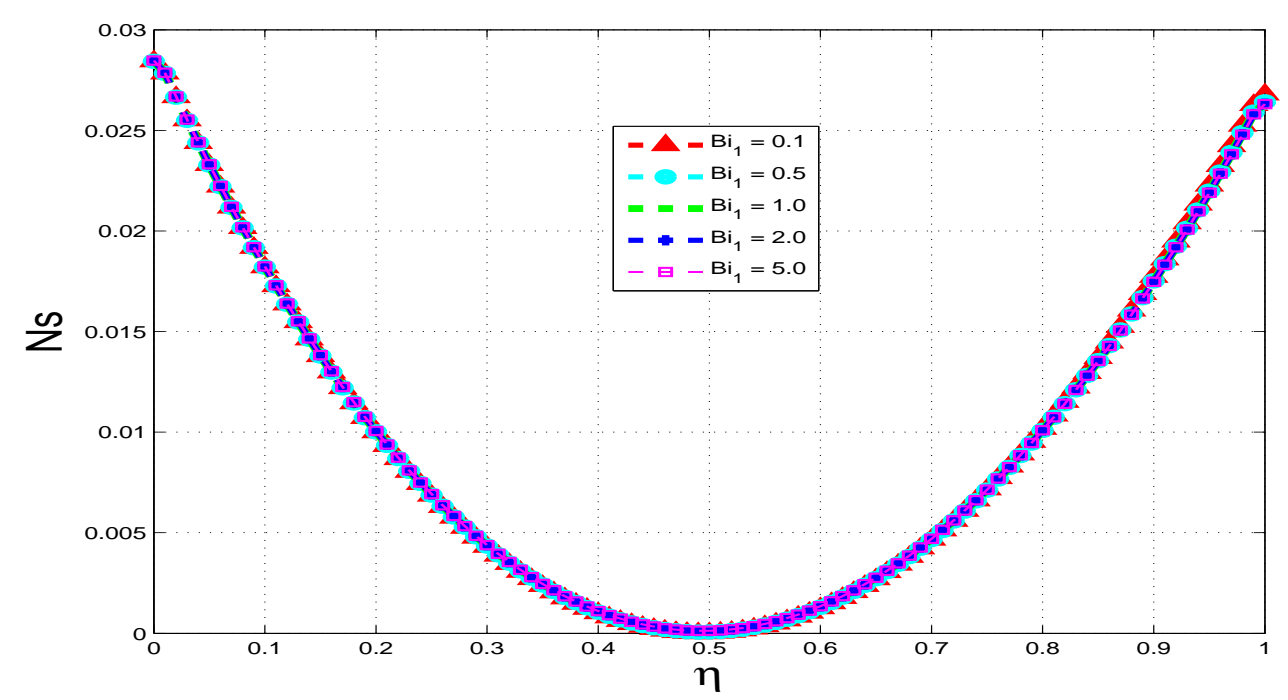

Figure 21. Variation of entropy generation rate with $\eta$ and $\mathrm{Bi}_{2}$.

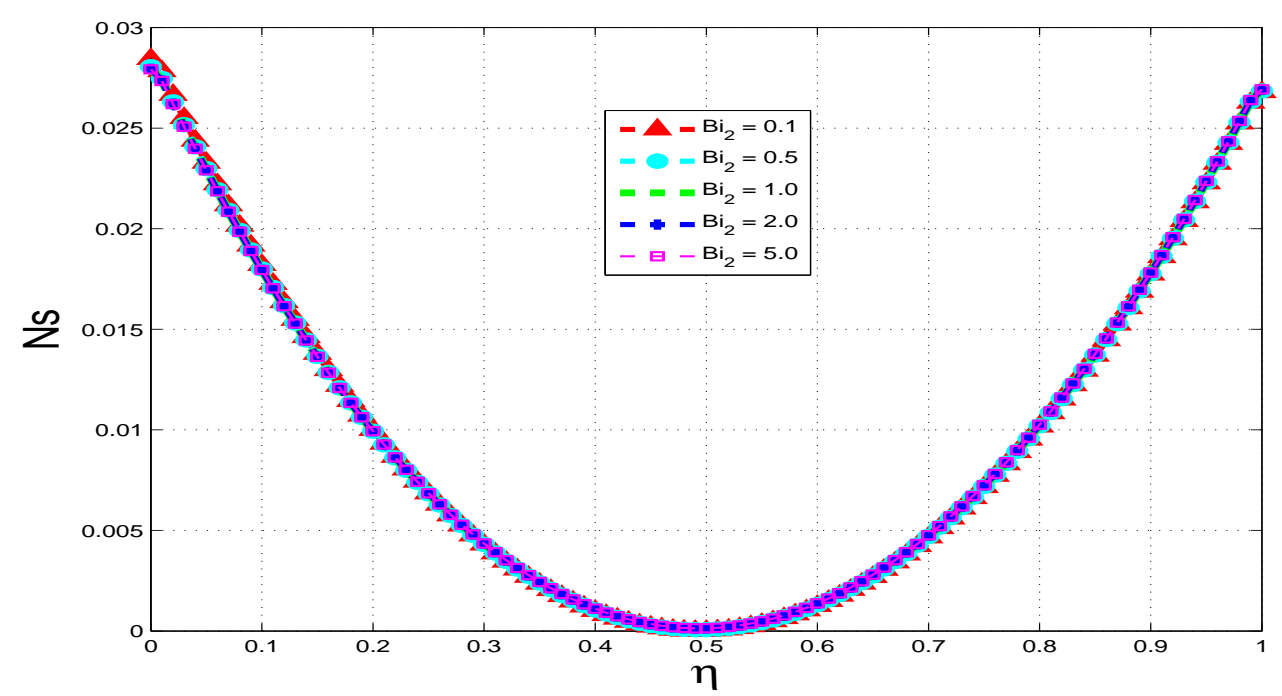

\subsection{Bejan Number}

In this section, we plot the Bejan number (Be) across the channel under varying parameter conditions. The analysis in this section is similar to that for the previous section with Ns now replaced by Be.

Figures 13-29 show as expected that parameters that increase the entropy generation rate will correspondingly decrease the Bejan number and vice versa.

Away from the wall (i.e., inside the main flow), the fluid friction with magnetic field irreversibility strongly dominates over heat transfer irreversibility. In the vicinity of the wall, the strength of the fluid parameters will determine which mode of irreversibility dominates over the other. 
Figure 22. Variation of Bejan number with $\eta$ and Re.

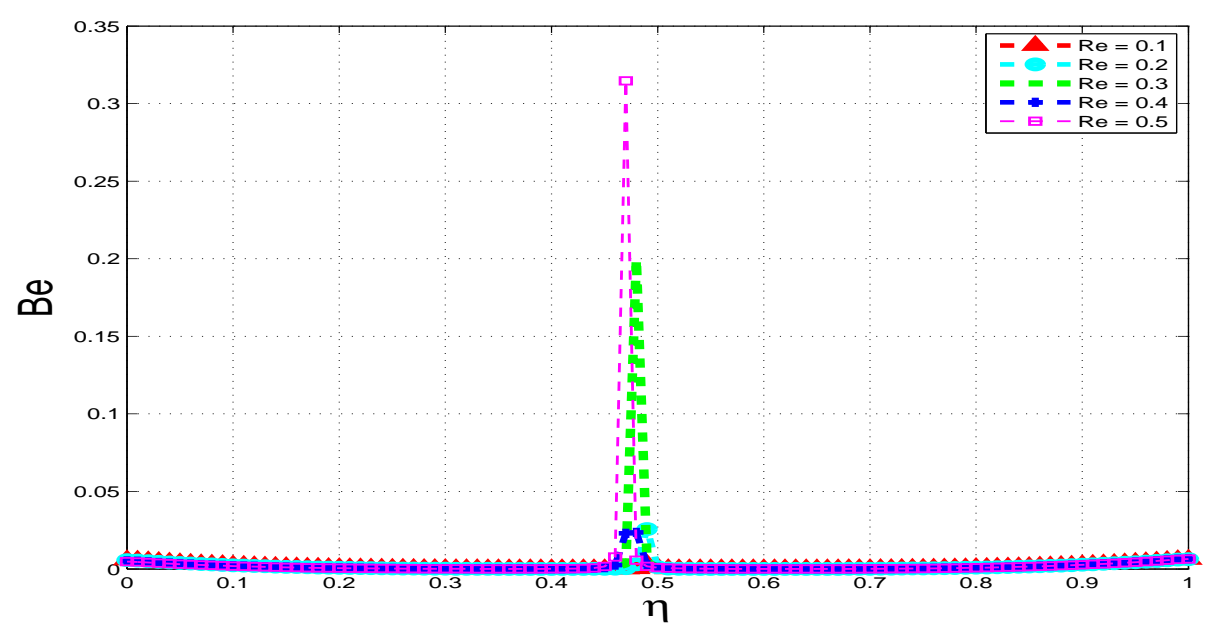

Figure 23. Variation of Bejan number with $\eta$ and Pr.

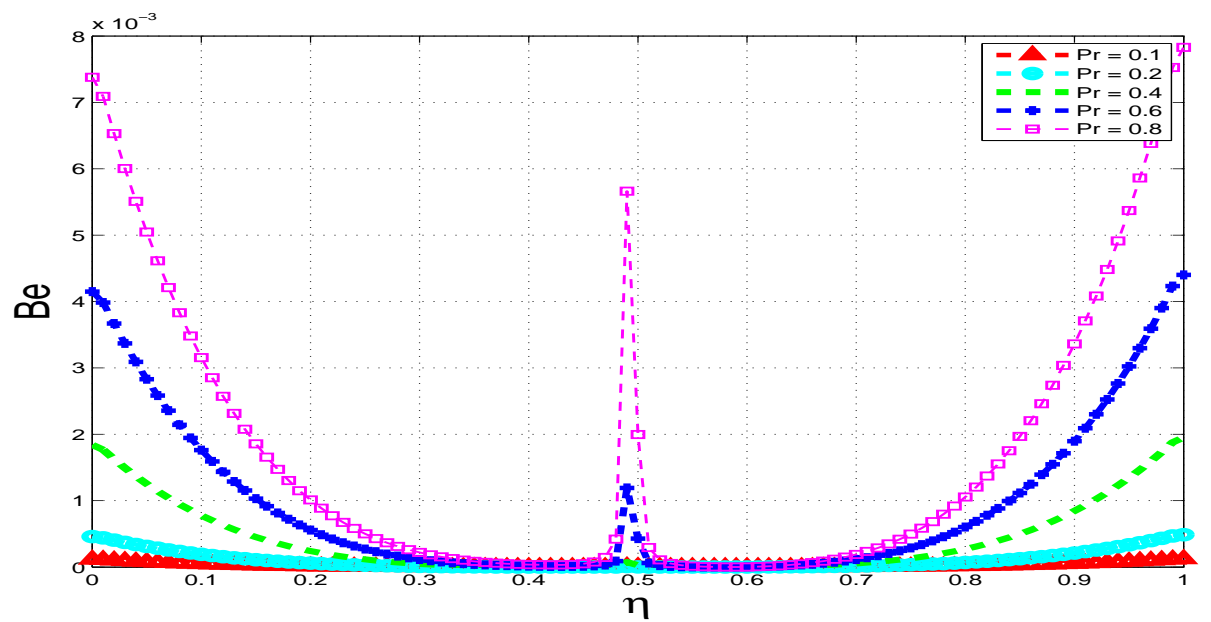

Figure 24. Variation of Bejan number with $\eta$ and $m$.

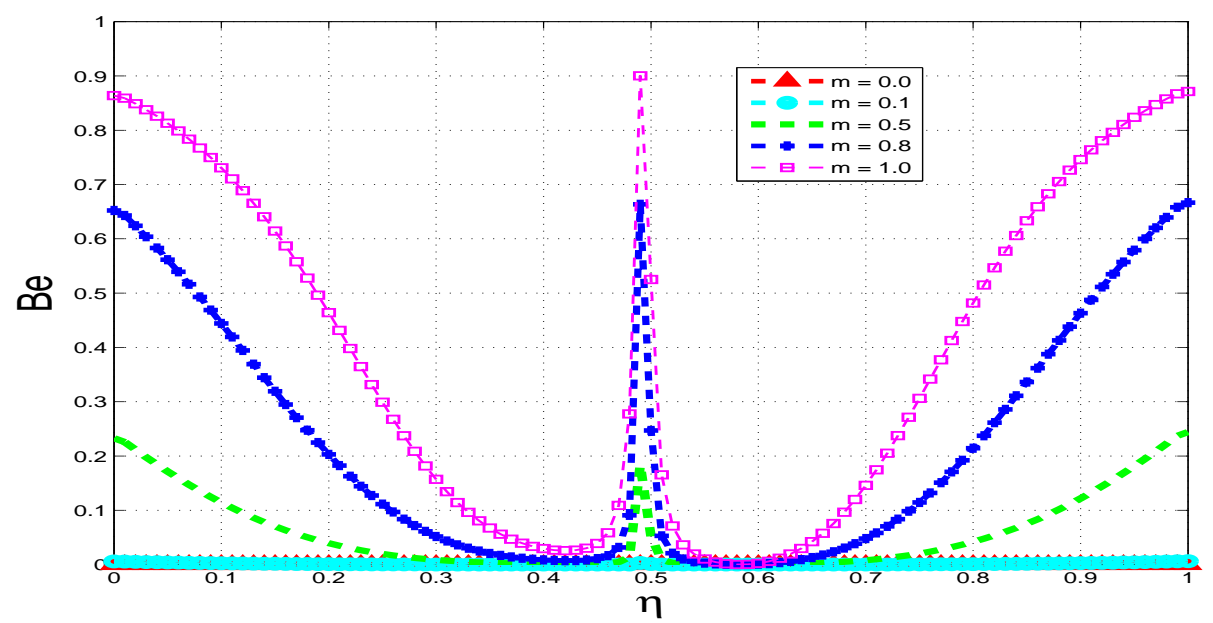


Figure 25. Variation of Bejan number with $\eta$ and $\operatorname{Br} \Omega^{-1}$.

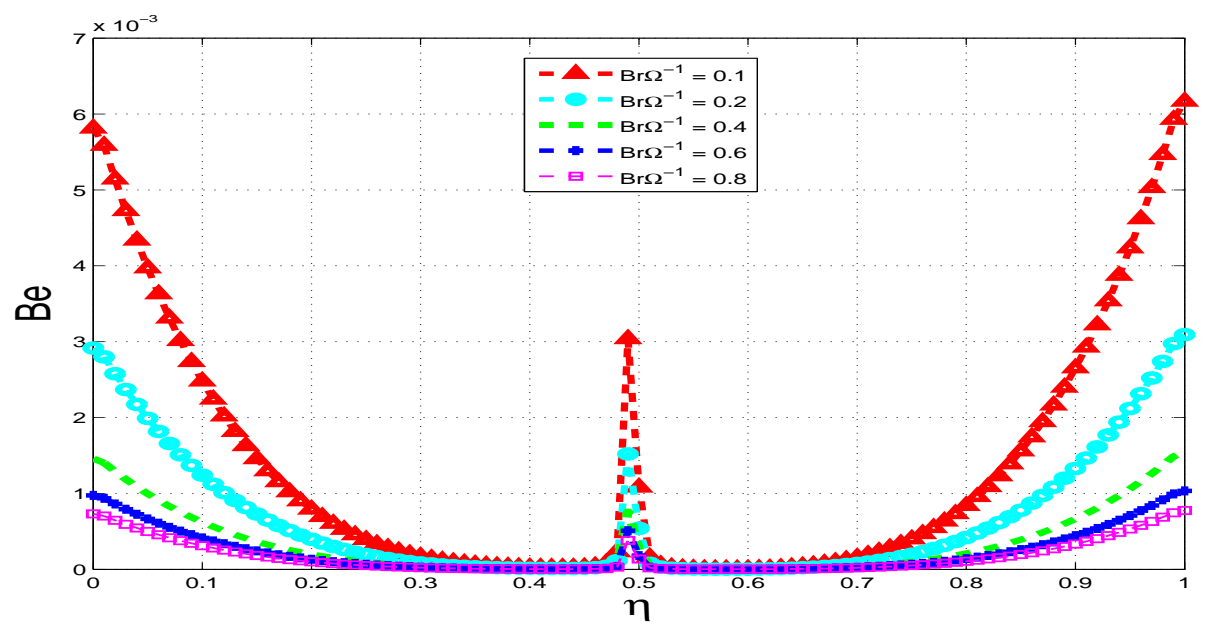

Figure 26. Variation of Bejan number with $\eta$ and $\lambda_{1}$.

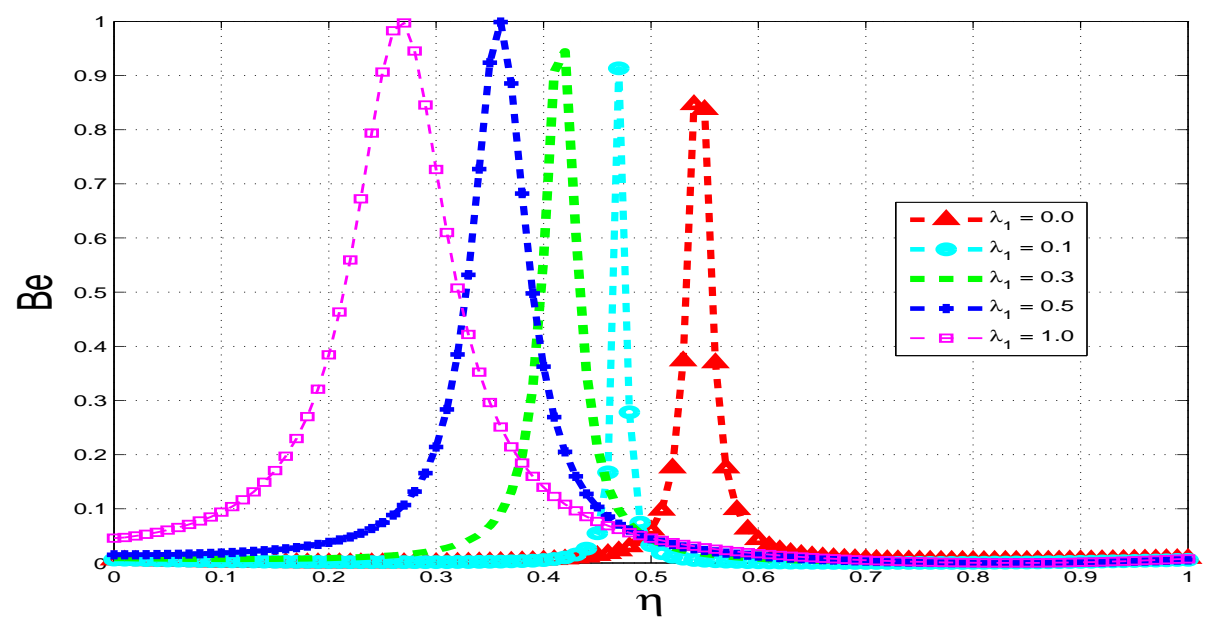

Figure 27. Variation of Bejan number with $\eta$ and $\lambda_{2}$.

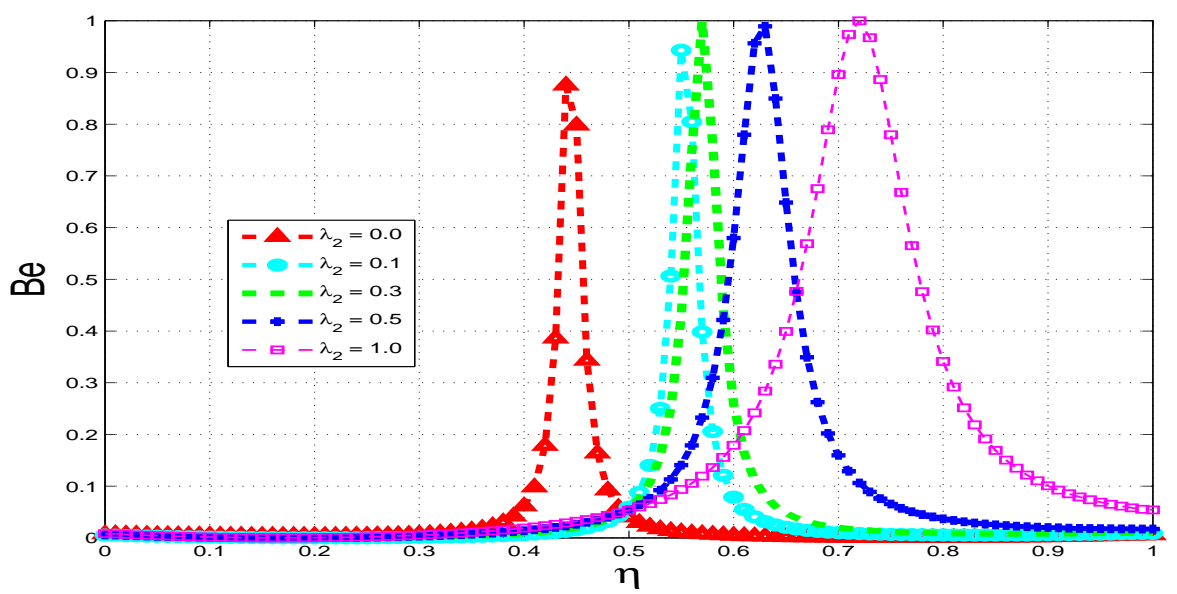


Figure 28. Variation of Bejan number with $\eta$ and $\mathrm{Bi}_{1}$.

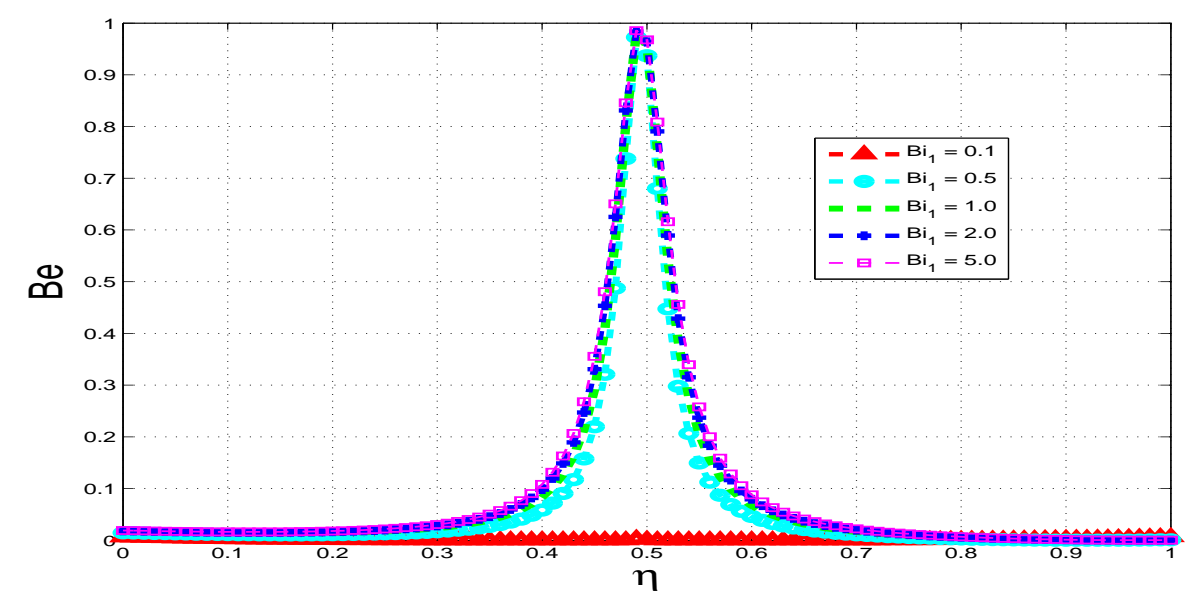

Figure 29. Variation of Bejan number with $\eta$ and $\mathrm{Bi}_{2}$.

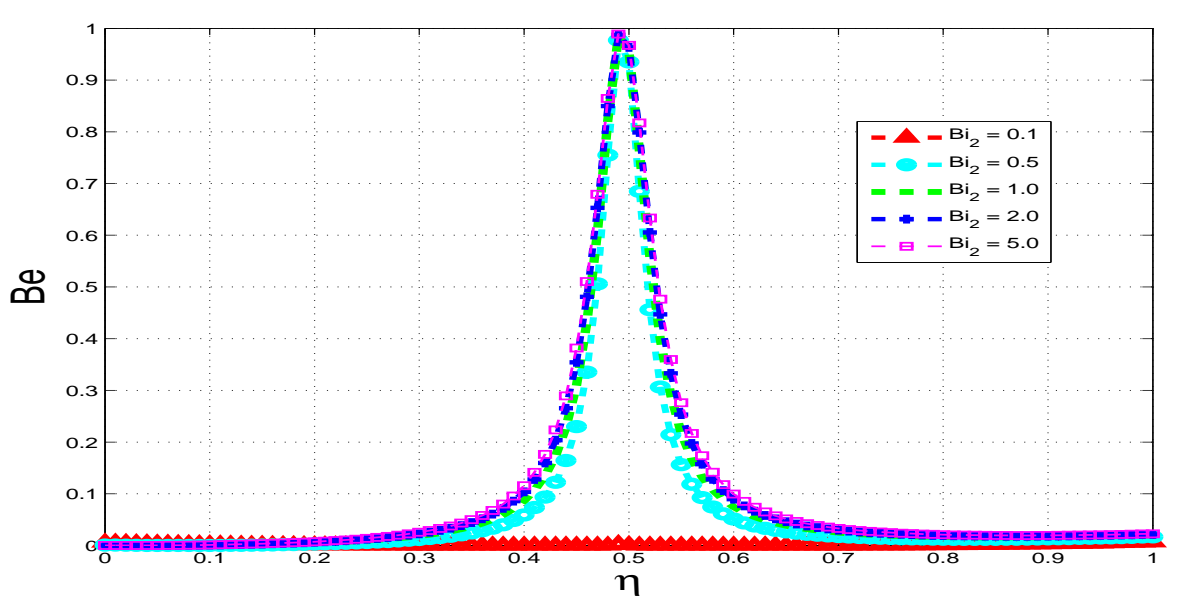

\section{Conclusions}

We computationally investigate the combined entropy generation rate in an unsteady porous channel flow with Navier slip subjected to asymmetrical convective boundary conditions. A major observation in the current work is the reduction in heat generation due to the presence of uniform suction/injection. We also notice that due to the nature of the coupling of the source terms, the fluid velocity and temperature either both increase or both decrease together. We have also demonstrated computationally that parameters that increase the entropy generation rate will correspondingly decrease the Bejan number and vice versa. In particular wall subjected to higher slip (i.e., lower viscosity) will have lower viscosities but higher Bejan numbers and vice versa.

\section{References}

1. Bejan, A. Second-law analysis in heat transfer and thermal design. Adv. Heat Transf. 1982, 15, $1-58$.

2. Bejan, A. Entropy Genaration Minimization; CRC: Boca Raton, NY, USA, 1996. 
3. Bejan, A. Fundamentals of exergy analysis, entropy generation minimization, and the generation of flow architecture. Int. J. Energy Res. 2002, 26, 545-565.

4. Wood, L.C. Thermodynamics of Fluid Systems; Oxford University Press: Oxford, UK, 1975.

5. Sahin, A.Z. A second law comparison for optimum shape of duct subjected to constant wall temperature and laminar flow. Heat Mass Transf. 1998, 33, 425-430.

6. Tasnim, S.H.; Mahmud, S. Entropy generation in a vertical concentric channel with temperature dependent viscosity. Int. Comm. Heat Mass Transf. 2002, 29, 907-918.

7. Mahmud, S.; Fraser, R.A. Thermodynamic analysis of flow and heat transfer inside channel with two parallel plates. Energy 2002, 2, 140-146.

8. Chinyoka, T. Suction-injection control of shear banding in non-isothermal and exothermic channel flow of johnson-segalman liquids. Trans. ASME J. Fluids Eng. 2011, 133, 071205.

9. Ireka, I.E.; Chinyoka, T. Non-isothermal flow of a Johnson-Segalman liquid in a lubricated pipe with wall slip. J. Non-Newton. Fluid Mechan. 2013, 192, 20-28.

10. Aziz, A. A similarity solution for laminar thermal boundary layer over a flat plate with a convective surface boundary condition. Commun. Nonlinear Sci. Numer. Simul. 2009, 14, 1064-1068.

11. Makinde, O.D.; Aziz, A. MHD mixed convection from a vertical plate embedded in a porous medium with a convective boundary condition. Int. J. Therm. Sci. 2010, 49, 1813-1820.

12. Makinde, O.D. Thermal ignition in a reactive viscous flow through a channel filled with a porous medium. Trans. ASME J. Heat Transf. 2006, 128, 601-604.

13. Makinde, O.D. Thermal stability of a reactive viscous flow through a porous-saturated channel with convective boundary conditions. Appl. Therm. Eng. 2009, 29, 1773-1777.

14. Siddiqui, A.M.; Mahmood, R.; Ghori, Q.K. Thin film flow of a third grade fluid on a moving belt by He's homotopy perturbation method. Int. J. Non-Linear Sci. Numer. Simul. 2006, 7, 1-8.

15. Massoudi, M.; Christe, I. Effects of variable viscosity and viscous dissipation on the flow of a third grade fluid in a pipe. Int. J. Non-Linear Mech. 1995, 30, 687-699.

16. Yurusoy, M.; Pakdemirli, M. Approximate analytical solutions for the flow of a third grade fluid in a pipe. Int. J. Non-Linear Mech. 2002, 37, 187-195.

17. Makinde, O.D.; Chinyoka, T. Numerical investigation of transient heat transfer to hydromagnetic channel flow with radiative heat and convective cooling. Commun. Nonlinear Sci. Numer. Simul. 2010, 15, 3919-3930.

18. Makinde, O.D.; Chinyoka, T. Transient analysis of pollutant dispersion in a cylindrical pipe with a nonlinear waste discharge concentration. Comput. Math. Appl. 2010, 60, 642-652.

19. Chinyoka, T. Computational dynamics of a thermally decomposable viscoelastic lubricant under shear. Trans. ASME J. Fluids Eng. 2008, 130, 121201.

20. Chinyoka, T. Poiseuille flow of reactive phan-thien-tanner liquids in 1D channel flow. Trans. ASME J. Heat Transf. 2010, 132, 111701.

(c) 2013 by the authors; licensee MDPI, Basel, Switzerland. This article is an open access article distributed under the terms and conditions of the Creative Commons Attribution license (http://creativecommons.org/licenses/by/3.0/). 